\title{
LINEAR SUPERGROUP ACTIONS. I: ON THE DEFINING PROPERTIES
}

\author{
OSCAR ADOLFO SÁNCHEZ VALENZUELA
}

\begin{abstract}
This paper studies the notions of linearity and bilinearity in the category of supermanifolds. Following the work begun by [OASV2], we deal with supermanifoldifications of supervector spaces. The $\mathbf{R}^{1 \mid 1}$-module operations are defined componentwise. The linearity and bilinearity properties are stated by requiring commutativity of some appropriate diagrams of supermanifold morphisms. It is proved that both linear and bilinear supermanifold morphisms are completely determined by their underlying continuous maps, which in turn have to be linear (resp., bilinear) in the usual sense. It is observed that whereas linear supermanifold morphisms are vector bundle maps, bilinear supermanifold morphisms are not. A natural generalization of the bilinear evaluation map $\operatorname{Hom}(V, W) \times V \rightarrow W((F, v) \mapsto F(v))$ is given and some applications pointing toward the notions of linear supergroup actions and adjoint and coadjoint actions are briefly discussed.
\end{abstract}

Introduction. This paper gives a generalization of the evaluation map we have at the level of supervector spaces and morphisms,

$$
\begin{gathered}
\Psi: \operatorname{Hom}(V, W) \times V \rightarrow W \\
(F, v) \mapsto F(v)
\end{gathered}
$$

to the realm of supermanifolds (for the sake of definiteness, we shall adopt the definition of supermanifold as given by [Manin]).

Thus, we first review those devices by which we may (functorially) assign supermanifolds and morphisms to supervector spaces and linear maps. In this direction we have, on the one hand, the correspondence already introduced in [Kostant] and [LeYtes],

$$
\begin{gathered}
\mathscr{O} b_{j} \text { jects(supervector spaces) } \rightarrow \mathscr{O} b_{\text {jects }} \text { (supermanifolds) } \\
V=V_{0} \oplus V_{1} \mapsto S V:=\left(V_{0},\left.C^{\infty}\right|_{V} \otimes \Lambda\left(V_{1}^{*}\right)\right)
\end{gathered}
$$

which can be easily supplemented with a corresponding assignment of morphisms

$$
\begin{gathered}
\text { Mor } p \text { hisms(supervector spaces) } \rightarrow \text { Mor } p \text { hisms(supermanifolds) } \\
(\operatorname{Hom}(V, W))_{0} \ni F \mapsto S F \in \operatorname{Mor}_{S}(S V, S W)
\end{gathered}
$$

so as to get a functor. This, however, can be understood in terms of classical differential geometry via vector bundles and bundle maps.

On the other hand, we have given arguments in [OASV2] suggesting that, in generalizing some of the notions of differential geometry to supergeometry, one should shift from $S V$ to $V_{S}:=S(V \oplus \Pi V)$ - the supermanifoldification of $V$.

Received by the editors March 18, 1987.

1980 Mathematics Subject Classification (1985 Revision). Primary 58A50. 
Following the rules of linear superalgebra developed in [OASV1], we provide a supermanifold morphism

$$
(\operatorname{Hom}(V, W))_{S} \times V_{S} \rightarrow W_{S}
$$

which generalizes (1).

After defining and studying the meaning of linearity and bilinearity in the supermanifold setting, we verify that (2) is an example of what we have called a superbilinear morphism. Furthermore, for each $F \in \operatorname{Hom}(V, W)$ (i.e., for each point in the underlying manifold of $\left.(\operatorname{Hom}(V, W))_{S}\right)$, the restricted morphism $V_{S} \rightarrow W_{S}$ that results from (2) is an example of what we have called a superlinear morphism. This was already pointed out in [OASV2].

We prove that a superlinear morphism, say $V_{S} \rightarrow W_{S}$, is completely determined by its underlying continuous map $V \rightarrow W$ and the latter has to be linear in the usual sense.

In complete analogy, we prove that - under certain symmetry assumptions - a superbilinear morphism

$$
U_{S} \times V_{S} \rightarrow W_{S}
$$

is also completely determined by its underlying continuous map $U \times V \rightarrow W$ and the latter has to be bilinear in the usual sense. The reconstruction process, however, is not as direct as it is in the superlinear case; this time it depends on the symmetry assumptions that specify the homogeneity behavior of (3) under scalar supermultiplication at the level of the second factor (i.e., $V_{S}$ ). An analogy can be established with maps $U \times V \rightarrow W$ of complex vector spaces which may be either C-linear or C-antilinear in the second entry. Besides, there is another important difference between the superlinear and superbilinear cases, consisting in the fact that, in general, superbilinear morphisms are not vector bundle maps.

Once the notions of linearity and bilinearity in the supermanifold context are clarified and an appropriate analog of the evaluation map (1) is defined, we obtain some insight about linear supergroup actions. Thus, for example, when we restrict (2) to the particular case $W=V=V_{0} \oplus V_{1}$, we may consider the supermanifolds

$$
\{S(\operatorname{Hom}(V, V))\}^{*} \text { and }\left\{\operatorname{Hom}(V, V)_{S}\right\}^{*}
$$

consisting of those subsupermanifolds of $S(\operatorname{Hom}(V, V))$ and $\operatorname{Hom}(V, V)_{S}$ respectively, described in local coordinates by the condition of having a nonzero Berezinian (cf. [Leĭtes]). Since a given matrix with entries in the augmented superalgebra $C^{\infty}(X) \otimes \bigwedge(Y) \rightarrow C^{\infty}(X)$ is invertible if and only if the corresponding matrix with entries in $C^{\infty}(X)$ is (cf. [Kostant] or [Leĭtes]), it follows that the underlying manifolds of the supergroups (4) must be $\mathrm{GL}\left(V_{0}\right) \times \mathrm{GL}\left(V_{1}\right)$ and $\mathrm{GL}(V)$, respectively. Their odd dimensions are also easy to deduce from this fact; they are $2 \operatorname{dim} V_{0} \operatorname{dim} V_{1}$ and $(\operatorname{dim} V)^{2}$, respectively. Moreover, these supergroups act-in the sense of (2)--on the supermanifold $V_{S}$.

There is also a possibility of obtaining-along classical lines--the notion of superadjoint and supercoadjoint actions originally defined in [Kostant]. The starting point would be to give ourselves a Lie superalgebra $\mathfrak{g}=\mathfrak{g}_{0} \oplus \mathfrak{g}_{1}$ and to consider its supermanifoldification $\mathfrak{g}_{S}$. Then, we use the Lie superbracket bilinear map $\mathfrak{g} \times \mathfrak{g} \rightarrow \mathfrak{g}$, together with some symmetry assumption, to construct a superbilinear 
bracket

$$
[,]: \mathfrak{g}_{S} \times \mathfrak{g}_{S} \rightarrow \mathfrak{g}_{S} .
$$

Once a supermanifold generalization of the Lie superbracket is defined, there is a unique manner of making sense of the superadjoint representation

$$
\mathfrak{a} \mathfrak{d}: \mathfrak{g}_{S} \rightarrow \operatorname{End}\left(\mathfrak{g}_{S}\right) \simeq(\operatorname{End} \mathfrak{g})_{S}
$$

and hence of the superadjoint action by means of the following diagram:

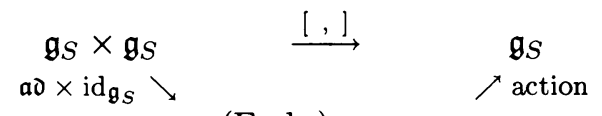

$(\text { End } \mathfrak{g})_{S} \times \mathfrak{g}_{S}$

where the action morphism is the one obtained via (2) with $V=\mathfrak{g}=W$.

Furthermore, (2) is also useful in generalizing the pairing between a supervector space and its dual (i.e., taking $W=\mathbf{R}$ ), and we may apply the resulting construction to the Lie superalgebra $V=\mathfrak{g}$ and obtain the superbilinear pairing

$$
\langle,\rangle:\left(\mathfrak{g}^{*}\right)_{S} \times \mathfrak{g}_{S} \rightarrow \mathbf{R}_{S} .
$$

Definition of the coadjoint representation is then obtained upon requiring the commutativity of the appropriate diagram involving the supermanifold morphisms (5), (6) and (8) as well as the actions gotten via (2). Explicit constructions and examples will be given in a forthcoming paper.

1. Supervector spaces and supermanifolds. Let $V=V_{0} \oplus V_{1}$ be a given $(m, n)$-dimensional supervector space (over $\mathbf{R}$ ). According to [Kostant] (see also [Leites]), there is an $(m, n)$-dimensional affine supermanifold that is naturally assigned to $V=V_{0} \oplus V_{1}$; namely, the supermanifold

$$
S V:=\left(V_{0},\left.C^{\infty}\right|_{V} \otimes \bigwedge\left(V_{1}^{*}\right)\right)
$$

We may think of the rule

$$
V \mapsto S V
$$

as a correspondence between objects in the category of supervector spaces and objects in the category of supermanifolds. Then, it is only natural to ask ourselves if there is also a natural assignment of morphisms; that is, a correspondence

$$
\operatorname{Hom}(V, W) \ni F \mapsto S F \in \operatorname{Mor}(S V, S W) .
$$

The answer to this question is: yes, when we restrict ourselves to $F$ 's in the even subspace $(\operatorname{Hom}(V, W))_{0}$. In fact, it is well known that the supermanifold morphism

$$
S F: S V \rightarrow S W
$$

is completely determined by the superalgebra morphism

$$
(S F)^{\#}: C^{\infty}\left(W_{0}\right) \otimes \bigwedge\left(W_{1}^{*}\right) \rightarrow C^{\infty}\left(V_{0}\right) \otimes \bigwedge\left(V_{1}^{*}\right)
$$

(cf. [Kostant], [Lertes]) and the latter can be constructed from $F$ as follows: first, we may take advantage of the existing functorial correspondence between vector spaces and their duals:

$$
\begin{aligned}
V & \mapsto V^{*}=\operatorname{Hom}(V, \mathbf{R}), \\
\operatorname{Hom}(V, W) \ni F & \mapsto F^{*} \in \operatorname{Hom}\left(W^{*}, V^{*}\right) .
\end{aligned}
$$


Then, the condition $F \in(\operatorname{Hom}(V, W))_{0}$ implies the pair of conditions

$$
F^{*}\left(W_{0}^{*}\right) \subset V_{0}^{*} \text { and } F^{*}\left(W_{1}^{*}\right) \subset V_{1}^{*} .
$$

Since for any vector space $U$ we have

$$
\begin{gathered}
U^{*}=\operatorname{Hom}(U, \mathbf{R}) \subset \operatorname{Sym}(U) \subset C^{\infty}(U), \\
U^{*} \subset \Lambda\left(U^{*}\right),
\end{gathered}
$$

the conditions (1.7) can be used to define the restrictions

$$
\left.(S F)^{\#}\right|_{W_{0}^{*} \otimes\{1\}}=\left.\left.F^{*}\right|_{W_{0}^{*}} \otimes \mathrm{id}\right|_{\{1\}}: W_{0}^{*} \otimes\{1\} \rightarrow V_{0}^{*} \otimes\{1\},
$$

and

$$
\left.(S F)^{\#}\right|_{\{1\} \otimes W_{1}^{*}}=\left.\left.\mathrm{id}\right|_{\{1\}} \otimes F^{*}\right|_{W_{1}^{*}}:\{1\} \otimes W_{1}^{*} \rightarrow\{1\} \otimes V_{1}^{*} .
$$

Then, use the fact that (1.9a) can be extended uniquely to an algebra homomorphism $\operatorname{Sym}\left(W_{0}\right) \otimes\{1\} \rightarrow \operatorname{Sym}\left(V_{0}\right) \otimes\{1\}$ and, since any $C^{\infty}$ function in $W_{0}$ can be approximated by polynomials (i.e., by elements of the subalgebra $\operatorname{Sym}\left(W_{0}\right)$ ), this homomorphism can be further extended to

$$
C^{\infty}\left(W_{0}\right) \otimes\{1\} \rightarrow C^{\infty}\left(V_{0}\right) \otimes\{1\} .
$$

Similarly, (1.9b) has a unique extension to a $\mathbf{Z}$-graded algebra homomorphism

$$
\{1\} \otimes \Lambda\left(W_{1}^{*}\right) \rightarrow\{1\} \otimes \Lambda\left(V_{1}^{*}\right),
$$

and there is only one way of putting these two homomorphisms together so as to obtain the desired superalgebra morphism

$$
(S F)^{\#}: C^{\infty}\left(W_{0}\right) \otimes \Lambda\left(W_{1}^{*}\right) \rightarrow C^{\infty}\left(V_{0}\right) \otimes \Lambda\left(V_{1}^{*}\right) .
$$

REMARK. One notes that the assignment

$$
\begin{gathered}
\mathscr{O} b_{j} \text { ects (supervector spaces) } \rightarrow \mathscr{O} l_{\text {jects }} \text { (supermanifolds), } \\
V=V_{0} \oplus V_{1} \mapsto S V:=\left(V_{0},\left.C^{\infty}\right|_{V} \otimes \Lambda\left(V_{1}^{*}\right)\right),
\end{gathered}
$$

together with the corresponding assignment of morphisms

$$
\text { Mor } p \text { hisms (supervector spaces) } \rightarrow \text { Mor } p \text { hisms(supermanifolds), }
$$

$$
(\operatorname{Hom}(V, W))_{0} \ni F \mapsto S F \in \operatorname{Mor}_{S}(S V, S W),
$$

does not really leave the category of vector bundles and morphisms. In fact, the supermanifold $S V$ is just the exterior algebra bundle of the (trivial) vector bundle $V_{0} \oplus V_{1} \rightarrow V_{0}$ given via projection onto the first factor, and the supermanifold morphism $S F$ is just a morphism of vector bundles. We shall have occasion of finding less trivial supermanifold morphisms throughout this work, though.

2. Linear supergroup actions. Let $V=V_{0} \oplus V_{1}$ and $W=W_{0} \oplus W_{1}$ be finite dimensional supervector spaces. Then $\operatorname{Hom}(V, W)$ is again a finite dimensional supervector space and we may consider the supermanifold $S(\operatorname{Hom}(V, W))$. Just as there is an action (or evaluation) map

$$
\begin{gathered}
\Psi: \operatorname{Hom}(V, W) \times V \rightarrow W \\
(F, v) \mapsto F(v)
\end{gathered}
$$


one would be naturally led to the question of what could be its supermanifold counterpart; i.e., is there a "reasonable" supermanifold morphism

$$
\text { " } S \Psi ": S(\operatorname{Hom}(V, W)) \times S V \rightarrow S W
$$

that we might call the action? What comes first to one's mind is a morphism that restricts, in the appropriate sense, to the evaluation map $\Psi$ and with a functorial behavior under compositions.

On these grounds, however, a supermanifold morphism like (2.2) is not entirely satisfactory. The reason is that the underlying manifold of $S(\operatorname{Hom}(V, W))$ consists of

$$
(\operatorname{Hom}(V, W))_{0}=\operatorname{Hom}\left(V_{0}, W_{0}\right) \oplus \operatorname{Hom}\left(V_{1}, W_{1}\right) \simeq \operatorname{Hom}\left(V_{0}, W_{0}\right) \times \operatorname{Hom}\left(V_{1}, W_{1}\right),
$$

whereas the underlying manifolds of $S V$ and $S W$ are $V_{0}$ and $W_{0}$, respectively. In particular, no matter what the actual supermanifold morphism (2.2) is, its underlying continuous map would have to be of the form

$$
\left(\operatorname{Hom}\left(V_{0}, W_{0}\right) \times \operatorname{Hom}\left(V_{1}, W_{1}\right)\right) \times V_{0} \rightarrow W_{0} .
$$

For this to be natural and have to do with the evaluation $\operatorname{map} \Psi, \operatorname{Hom}\left(V_{1}, W_{1}\right)$ would have to play no role in it.

There is one easy way out of this problem, which consists of considering $S(V \oplus \Pi V)$ and $S(W \oplus \Pi W)$ rather than just $S V$ and $S W$; $\Pi$ being the so-called change of parity "functor" (see [Leǐtes]). The supermanifold $S(V \oplus \Pi V)$ was called in [OASV2] the supermanifoldification of $V$ and was denoted by $V_{S}$.

Note. Let us briefly recall that if $V=V_{0} \oplus V_{1}$ is a supervector space, then, $\Pi V=(\Pi V)_{0} \oplus(\Pi V)_{1}$ is again a supervector space, with

$$
(\Pi V)_{0}=V_{1} \text { and }(\Pi V)_{1}=V_{0} \text {. }
$$

Furthermore, there is a natural odd isomorphism

$$
\pi: V \rightarrow \Pi V
$$

which allows us to identify $\Pi V$ with $\pi(V)$ and hence $V$ itself with $\Pi \Pi V$, as $\pi^{2}$ is the identity. In fact, such a $\pi$ is defined via the conditions

(i) for each homogeneous $v \in V, \pi v$ is homogeneous and $|\pi v|=|v|+1$;

(ii) in the sense of set theory, $\pi v=v$.

Thus, instead of (2.2), one would attempt an action morphism of the form

$$
S \Psi: S(\operatorname{Hom}(V, W)) \times V_{S} \rightarrow W_{S}
$$

which at least has the possibility of producing an underlying continuous map of the form

$$
\left(\operatorname{Hom}\left(V_{0}, W_{0}\right) \oplus \operatorname{Hom}\left(V_{1}, W_{1}\right)\right) \times\left(V_{0} \oplus V_{1}\right) \rightarrow W_{0} \oplus W_{1} .
$$

In this case, we know that $\operatorname{Hom}\left(V_{0}, W_{0}\right)$ acts on $V_{0}$ and $\operatorname{Hom}\left(V_{1}, W_{1}\right)$ acts on $V_{1}$ and therefore we have a chance of recovering the action of $(\operatorname{Hom}(V, W))_{0}$ on $V$. But this is not exactly (2.1), yet. If we insist on getting (2.1) as the underlying continuous map of a supermanifold morphism, we are led to a morphism of the type

$$
\Psi_{S}: \operatorname{Hom}(V, W)_{S} \times V_{S} \rightarrow W_{S}
$$


Now, if we are going to follow classical analogies, appropriate notions of linearity and bilinearity must be introduced. In fact, at the classical level, the map

$$
\Psi: \operatorname{Hom}(V, W) \times V \rightarrow W
$$

$$
(F, v) \mapsto F(v)
$$

yields, for each $F \in \operatorname{Hom}(V, W)$, a linear map

$$
\begin{gathered}
\Psi_{F}: V \rightarrow W, \\
v \mapsto F(v),
\end{gathered}
$$

whereas the map $\Psi$ itself is bilinear. $\S \S 3$ and 4 below will be devoted to the characterization of linearity and bilinearity in the supermanifold setting. In the meantime, however, we can find supermanifold morphisms like (2.2), (2.7) and (2.9) by a heuristic use of the methods of $\S 1$ : we note first of all that, associated to the bilinear map (2.1), there is a linear map

$$
\begin{gathered}
\Phi: \operatorname{Hom}(V, W) \otimes V \rightarrow W, \\
F \otimes v \mapsto F(v),
\end{gathered}
$$

whose corresponding supermanifold morphism

$$
S \Phi: S(\operatorname{Hom}(V, W) \otimes V) \rightarrow S W
$$

may be constructed as in $\S 1$. In fact, note that the map $\Phi$ is homogeneous of degree zero, for

$$
|\Phi|+|F|+|v|=|\Phi|+|F \otimes v|=|\Phi(F \otimes v)|=|F(v)|=|F|+|v| .
$$

Now, even though our approach in $\S 1$ to the morphism $S \Phi$ was independent of any use of coordinates, let us try here to give an explicit description of it in terms of a special set of linear coordinates; namely, assume that we are given graded bases

$$
\begin{gathered}
\left\{v_{i} ; s_{I}: i \in\left\{1,2, \ldots, \operatorname{dim} V_{0}\right\}, I \in\left\{1,2, \ldots, \operatorname{dim} V_{1}\right\}\right\}, \\
\left\{w_{a} ; t_{A}: a \in\left\{1,2, \ldots, \operatorname{dim} W_{0}\right\}, A \in\left\{1,2, \ldots, \operatorname{dim} W_{1}\right\}\right\}
\end{gathered}
$$

of $V$ and $W$. Then, their corresponding dual bases

$$
\begin{gathered}
\left\{x^{i} ; \xi^{I}: i \in\left\{1,2, \ldots, \operatorname{dim} V_{0}\right\}, I \in\left\{1,2, \ldots, \operatorname{dim} V_{1}\right\}\right\}, \\
\left\{y^{a} ; \varsigma^{A}: a \in\left\{1,2, \ldots, \operatorname{dim} W_{0}\right\}, A \in\left\{1,2, \ldots, \operatorname{dim} W_{1}\right\}\right\}
\end{gathered}
$$

give (global) coordinate systems on the supermanifolds $S V$ and $S W$, respectively. Let us assume that $\operatorname{Hom}(V, W)$ has been equipped with the basis

$$
\left\{E_{b j}, F_{B J} ; P_{b J}, Q_{B j}\right\}
$$

for which

$$
\begin{aligned}
& \operatorname{Hom}\left(V_{0}, W_{0}\right) \simeq \operatorname{span}\left\{E_{b j}: E_{b j}\left(v_{i}\right)=\delta_{i j} w_{b}, E_{b j}\left(s_{I}\right)=0\right\}, \\
& \operatorname{Hom}\left(V_{1}, W_{1}\right) \simeq \operatorname{span}\left\{F_{B J}: F_{B J}\left(v_{i}\right)=0, F_{B J}\left(s_{I}\right)=\delta_{I J} t_{B}\right\}, \\
& \operatorname{Hom}\left(V_{1}, W_{0}\right) \simeq \operatorname{span}\left\{P_{b J}: P_{b J}\left(v_{i}\right)=0, P_{b J}\left(s_{I}\right)=\delta_{I J} w_{b}\right\}, \\
& \operatorname{Hom}\left(V_{0}, W_{1}\right) \simeq \operatorname{span}\left\{Q_{B j}: Q_{B j}\left(v_{i}\right)=\delta_{i j} t_{B}, Q_{B j}\left(s_{I}\right)=0\right\},
\end{aligned}
$$

and let us denote by $\left\{A^{b j}, D^{B J} ; \Gamma^{b J}, \Theta^{B j}\right\}$ the corresponding dual basis. This gives a (global) coordinate system on $S(\operatorname{Hom}(V, W))$. It is easy to see then that (2.17) $\left\{A^{b j} \otimes x^{i}, D^{B J} \otimes x^{i}, \Gamma^{b J} \otimes \xi^{I}, \Theta^{B j} \otimes \xi^{I} ; A^{b j} \otimes \xi^{I}, D^{B J} \otimes \xi^{I}, \Gamma^{b J} \otimes x^{i}, \Theta^{B j} \otimes x^{i}\right\}$ is a (global) coordinate system on the supermanifold $S(\operatorname{Hom}(V, W) \otimes V)$. 
2.1 PROPOSITION. The effect of the superalgebra morphism

$(S \Phi)^{\#}: C^{\infty}\left(W_{0}\right) \otimes \bigwedge\left(W_{1}^{*}\right) \rightarrow C^{\infty}\left((\operatorname{Hom}(V, W) \otimes V)_{0}\right) \otimes \bigwedge\left((\operatorname{Hom}(V, W) \otimes V)_{1}^{*}\right)$

with respect to the coordinate systems above is given by

$$
\begin{aligned}
& (S \Phi)^{\#} y^{b}=\sum_{j} A^{b j} \otimes x^{j}-\sum_{J} \Gamma^{b J} \otimes \xi^{J}, \\
& (S \Phi)^{\#} \zeta^{B}=\sum_{j} \Theta^{B j} \otimes x^{j}+\sum_{J} D^{B J} \otimes \xi^{J} .
\end{aligned}
$$

PROOF. We only have to compute the effect of the dual map

$$
\Phi^{*} \in \operatorname{Hom}\left(W^{*},(\operatorname{Hom}(V, W) \otimes V)^{*}\right)
$$

with respect to the given basis. This can be done by writing out $\Phi^{*} y^{b}$ and $\Phi^{*} \varsigma^{B}$ as linear combinations of the basis (2.17) and computing the values $\left\langle F \otimes v, \Phi^{*} y^{b}\right\rangle$ and $\left\langle F \otimes v, \Phi^{*} \varsigma^{B}\right\rangle$ in two different ways: on the one hand, since $|\Phi|=0$, the definition of $\Phi^{*}$ says that, for homogeneous decomposable elements $F \otimes v$ of $\operatorname{Hom}(V, W) \otimes V$ and for any $\psi \in W^{*}$,

$$
\left\langle F \otimes v, \Phi^{*} \psi\right\rangle=\langle\Phi(F \otimes v), \psi\rangle=\langle F(v), \psi\rangle .
$$

On the other hand, since $\Phi^{*} \psi$ is a linear combination of elements of the form $\varepsilon \otimes \chi \in(\operatorname{Hom}(V W))^{*} \otimes V^{*} \simeq(\operatorname{Hom}(V, W) \otimes V)^{*}$, the values $\left\langle F \otimes v, \Phi^{*} \psi\right\rangle$ give rise to expressions of the type $\langle F \otimes v, \varepsilon \otimes \chi\rangle$; these duality relations are then computed in the graded sense (cf. [OASV1]):

$$
\langle F \otimes v, \varepsilon \otimes \chi\rangle=(-1)^{|v||\varepsilon|}\langle F, \varepsilon\rangle\langle v, \chi\rangle
$$

where $v$ and $\varepsilon$ are assumed to be homogeneous. By letting $F$ and $v$ run through $\left\{E_{b j}, F_{B J} ; P_{b J}, Q_{B j}\right\}$ and $\left\{v_{i} ; s_{I}\right\}$, respectively, one immediately shows that indeed, $\Phi^{*} y^{b}$ and $\Phi^{*} \zeta^{B}$ are given as in the statement.

The heuristic part of the argument that leads us to the supermanifold morphism (2.2) consists of replacing the tensor products in the statement above by multiplication in the superalgebra

$$
C^{\infty}\left((\operatorname{Hom}(V, W) \times V)_{0}\right) \otimes \bigwedge\left((\operatorname{Hom}(V, W) \times V)_{1}^{*}\right)
$$

with the prefix $p_{1}^{\#}$ (resp., $p_{2}^{\#}$ ) in front of the coordinates $\left\{A^{b j}, D^{B J} ; \Gamma^{b J}, \Theta^{B j}\right\}$ (resp., $\left.\left\{x^{i} ; \xi^{I}\right\}\right), p_{1}$ and $p_{2}$ being the projection morphisms

$$
\begin{gathered}
p_{1}: S(\operatorname{Hom}(V, W)) \times S V \rightarrow S(\operatorname{Hom}(V, W)), \\
p_{2}: S(\operatorname{Hom}(V, W)) \times S V \rightarrow S V
\end{gathered}
$$

onto the first and second factors, respectively. Thus, a coordinate expression for the morphism (2.2) is given by

$$
\begin{aligned}
& \left(" S \Psi " \Psi^{\#} y^{b}=\sum_{j}\left(p_{1}^{\#} A^{b j}\right)\left(p_{2}^{\#} x^{j}\right)-\sum_{J}\left(p_{1}^{\#} \Gamma^{b J}\right)\left(p_{2}^{\#} \xi^{J}\right)\right. \\
& (" S \Psi "){ }^{\#} \varsigma^{B}=\sum_{j}\left(p_{2}^{\#} \Theta^{B j}\right)\left(p_{2}^{\#} x^{j}\right)+\sum_{J}\left(p_{2}^{\#} D^{B J}\right)\left(p_{2}^{\#} \xi^{J}\right)
\end{aligned}
$$


which can be rewritten in matrix form as

$$
(" S \Psi "))^{\#}\left(\begin{array}{c}
y^{b} \\
\varsigma^{B}
\end{array}\right)=p_{1}^{\#}\left(\begin{array}{cc}
A^{b j} & \Gamma^{b J} \\
\Theta^{B j} & D^{B J}
\end{array}\right) \cdot p_{2}^{\#}\left(\begin{array}{c}
x^{j} \\
\xi^{J}
\end{array}\right) .
$$

We remark that the strange minus sign that appears in (2.19) is important in order to accomplish the last step (cf. [OASV1]).

We may proceed analogously and get coordinate expressions for the morphisms (2.7) and (2.9), respectively. Thus, for example, a global coordinate system for the supermanifold $S\{(\operatorname{Hom}(V, W) \oplus \Pi \operatorname{Hom}(V, W)) \otimes(V \oplus \Pi V)\}$ is given by the following set of even coordinates:

$$
\begin{array}{llll}
A^{b j} \otimes x^{i} & A^{b j} \otimes \pi \xi^{I} & \pi \Theta^{B j} \otimes x^{i} & \pi \Theta^{B j} \otimes \pi \xi^{I} \\
D^{B J} \otimes x^{i} & D^{B J} \otimes \pi \xi^{I} & \pi \Gamma^{b J} \otimes x^{i} & \pi \Gamma^{b J} \otimes \pi \xi^{I} \\
\Theta^{B j} \otimes \xi^{I} & \Theta^{B j} \otimes \pi x^{i} & \pi A^{b j} \otimes \xi^{I} & \pi A^{b j} \otimes \pi x^{i} \\
\Gamma^{b J} \otimes \xi^{I} & \Gamma^{b J} \otimes \pi x^{i} & \pi D^{B J} \otimes \xi^{I} & \pi D^{B J} \otimes \pi x^{i}
\end{array}
$$

and odd coordinates:

$$
\begin{array}{llll}
A^{b j} \otimes \xi^{I} & A^{b j} \otimes \pi x^{i} & \pi \Theta^{B j} \otimes \xi^{I} & \pi \Theta^{B j} \otimes \pi x^{i} \\
D^{B J} \otimes \xi^{I} & D^{B J} \otimes \pi x^{i} & \pi \Gamma^{b J} \otimes \xi^{I} & \pi \Gamma^{b J} \otimes \pi x^{i} \\
\Theta^{B j} \otimes x^{i} & \Theta^{B j} \otimes \pi \xi^{I} & \pi A^{b j} \otimes x^{i} & \pi A^{b j} \otimes \pi \xi^{I} \\
\Gamma^{b J} \otimes x^{i} & \Gamma^{b J} \otimes \pi \xi^{I} & \pi D^{B J} \otimes x^{i} & \pi D^{B J} \otimes \pi \xi^{I} .
\end{array}
$$

(It is clear that a global coordinate system for the supermanifold

$$
S\{\operatorname{Hom}(V, W) \otimes(V \oplus \Pi V)\}
$$

is obtained by putting the last two rows in both sets equal to zero.) We then have the following.

2.2 PROPOSITION. With respect to the coordinate systems above,

(i) the effect of the superalgebra morphism

$$
\begin{gathered}
C^{\infty}\left((W \oplus \Pi W)_{0}\right) \otimes \Lambda\left((W \oplus \Pi W)_{1}^{*}\right) \\
C^{\infty}\left(\{\operatorname{Hom}(V, W) \otimes(V \oplus \Pi V)\}_{0}\right) \otimes \wedge\left(\{\operatorname{Hom}(V, W) \otimes(V \oplus \Pi V)\}_{1}^{*}\right),
\end{gathered}
$$

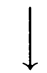

coming from the natural even extension

$$
\Phi: \operatorname{Hom}(V, W) \otimes(V \oplus \Pi V) \rightarrow W \oplus \Pi W
$$

of the linear map (2.12), is given by

$$
\begin{aligned}
& (S \Phi)^{\#} y^{b}=\sum_{j} A^{b j} \otimes x^{j}-\sum_{J} \Gamma^{b J} \otimes \xi^{J} \\
& (S \Phi)^{\#} \pi \zeta^{B}=-\sum_{j} \Theta^{B j} \otimes \pi x^{j}+\sum_{J} D^{B J} \otimes \pi \xi^{J} \\
& (S \Phi)^{\#} \pi y^{b}=\sum_{j} A^{b j} \otimes \pi x^{j}+\sum_{J} \Gamma^{b J} \otimes \pi \xi^{J} \\
& (S \Phi)^{\#} \zeta^{B}=\sum_{j} \Theta^{B j} \otimes x^{j}+\sum_{J} D^{B J} \otimes \xi^{J}
\end{aligned}
$$


(ii) the effect of the superalgebra morphism

$$
\begin{gathered}
C^{\infty}\left((W \oplus \Pi W)_{0}\right) \otimes \Lambda\left((W \oplus \Pi W)_{1}^{*}\right) \\
C^{\infty}\left(\{(\operatorname{Hom}(V, W) \oplus \Pi \operatorname{Hom}(V, W)) \otimes(V \oplus \Pi V)\}_{0}\right) \\
\otimes \wedge\left(\{(\operatorname{Hom}(V, W) \oplus \Pi \operatorname{Hom}(V, W)) \otimes(V \oplus \Pi V)\}_{1}^{*}\right)
\end{gathered}
$$

coming from the natural even extension

$$
\Phi:(\operatorname{Hom}(V, W) \oplus \Pi \operatorname{Hom}(V, W)) \otimes(V \oplus \Pi V) \rightarrow W \oplus \Pi W
$$

of the linear map (2.12a) is given by

$$
\begin{aligned}
(S \Phi)^{\#} y^{b}= & \sum_{j} A^{b j} \otimes x^{j}-\sum_{J} \Gamma^{b J} \otimes \xi^{J}+\sum_{j} \pi A^{b j} \otimes \pi x^{j}+\sum_{J} \pi \Gamma^{b J} \otimes \pi \xi^{J}, \\
(S \Phi)^{\#} \pi \zeta^{B}= & -\sum_{j} \Theta^{B j} \otimes \pi x^{j}+\sum_{J} D^{B J} \otimes \pi \xi^{J} \\
& +\sum_{j} \pi \Theta^{B j} \otimes x^{j}+\sum_{J} \pi D^{B J} \otimes \xi^{J}, \\
(S \Phi)^{\#} \pi y^{b}= & \sum_{j} A^{b j} \otimes \pi x^{j}+\sum_{J} \Gamma^{b J} \otimes \pi \xi^{J}+\sum_{j} \pi A^{b j} \otimes x^{j}-\sum_{J} \pi \Gamma^{b J} \otimes \xi^{J}, \\
(S \Phi)^{\#} S^{B}= & \sum_{j} \Theta^{B j} \otimes x^{j}+\sum_{J} D^{B J} \otimes \xi^{J}-\sum_{j} \pi \Theta^{B j} \otimes \pi x^{j}+\sum_{J} \pi D^{B J} \otimes \pi \xi^{J} .
\end{aligned}
$$

PROOF. In both cases the proof is completely analogous to that of 2.1 ; the only difference is that the computation of the linear maps (2.12a) and (2.12b) is to be carried out according to the following extension rules: for each homogeneous $F \in \operatorname{Hom}(V, W)$ and $v \in V$, we have

$$
\begin{aligned}
& \operatorname{Hom}(V, W) \otimes \Pi V \rightarrow \Pi W, \\
& F \otimes \pi v \mapsto(-1)^{|F|} \pi(F(v)) .
\end{aligned}
$$

It is then clear how to put (2.12) and (2.22) together to get what we have called the natural even extension, (2.12a), of (2.12). Similarly, we can extend (2.12a) itself and get (2.12b) as follows: first of all, for each $F \in \operatorname{Hom}(V, W)$ and $v \in V$, we may define

$$
\begin{gathered}
\Pi \operatorname{Hom}(V, W) \otimes V \rightarrow \Pi W \\
\pi F \otimes v \mapsto \pi(F(v))
\end{gathered}
$$

so as to have a natural isomorphism

$$
\Pi \operatorname{Hom}(V, W) \cong \operatorname{Hom}(V, \Pi W) \text {. }
$$

Then, note that an obvious combination of (2.22) and (2.23) with the fact that $\pi^{2}$ is the identity suggests to further set, for each homogeneous $F \in \operatorname{Hom}(V, W)$ and each $v \in V$,

$$
\begin{aligned}
& \Pi \operatorname{Hom}(V, W) \otimes \Pi V \rightarrow \Pi W \\
& \pi F \otimes \pi v \mapsto(-1)^{|\pi F|} F(v) .
\end{aligned}
$$


REMARK. In order to keep using the notation introduced in 2.1 , let us note that if $U$ is any supervector space, then, for each homogeneous $\chi \in \operatorname{Hom}(U, \mathbf{R})$ and each homogeneous $u \in U,\langle u, \chi\rangle=(-1)^{|u||\chi|} \chi(u)$. Therefore, in computing expressions of the form $\langle\pi u, \pi \chi\rangle$, we will have

$$
\langle\pi u, \pi \chi\rangle=(-1)^{|u|}\langle u, \chi\rangle,
$$

as the reader can easily verify.

Finally, as in the proof of 2.1 , the computations that lead to the statements are straightforward and are better left to the reader.

Just as before, we may obtain the supermanifold morphisms (2.7) and (2.9) after a formal replacement of the tensor products appearing in (i) and (ii) above by multiplication in the appropriate superalgebras and suitable projection morphisms as prefixes of the corresponding factors. We may also write the resulting expressions in matrix form as

$$
(S \Psi)^{\#}\left(\begin{array}{c}
y^{b}+\pi y^{b} \\
\varsigma^{B}+\pi \varsigma^{B}
\end{array}\right)=p_{1}^{\#}\left(\begin{array}{cc}
A^{b j} & \Gamma^{b \mathbf{J}} \\
\Theta^{B j} & D^{B J}
\end{array}\right) \cdot p_{2}^{\#}\left(\begin{array}{c}
x^{j}+\pi x^{j} \\
\xi^{J}+\pi \xi^{J}
\end{array}\right)
$$

and

$$
\left(\Psi_{S}\right)^{\#}\left(\begin{array}{c}
y^{b}+\pi y^{b} \\
\varsigma^{B}+\pi \varsigma^{B}
\end{array}\right)=p_{1}^{\#}\left(\begin{array}{cc}
A^{b j}+\pi A^{b j} & \Gamma^{b J}+\pi \Gamma^{b J} \\
\Theta^{B j}+\pi \Theta^{B j} & D^{B J}+\pi D^{B J}
\end{array}\right) \cdot p_{2}^{\#}\left(\begin{array}{c}
x^{j}+\pi x^{j} \\
\xi^{J}+\pi \xi^{J}
\end{array}\right)
$$

respectively, for the minus signs appearing in (i) and (ii) are precisely those required by the rules of linear superalgebra to accomplish these steps (cf. [OASV2]).

REMARK. We shall see in $\S 4$ that the morphisms (2.7) and (2.9) thus obtained from 2.2 are examples of superbilinear morphisms. We have already used the coordinate expressions of these morphisms in the various constructions encountered in our work with supervector bundles [OASV2].

We would like to close this section by noting that if we restrict (2.7) and (2.9) to the special case $V=W$, we get at least two supergroups in sight with a more or less expected geometric (and algebraic, as will follow from §3) meaning: namely, the supermanifolds

$$
\{S(\operatorname{Hom}(V, V))\}^{*} \text { and }\left\{\operatorname{Hom}(V, V)_{S}\right\}^{*}
$$

consisting of those subsupermanifolds of $S(\operatorname{Hom}(V, V))$ and $\operatorname{Hom}(V, V)_{S}$ respectively described in local coordinates by the condition of having a nonzero Berezinian (cf. [Leǐtes]). Since a given matrix with entries in the augmented superalgebra $C^{\infty}(X) \otimes \bigwedge(Y) \rightarrow C^{\infty}(X)$ is invertible if and only if the corresponding matrix with entries in $C^{\infty}(X)$ is (cf. [Kostant] or [Leřtes]), it follows that the underlying manifolds of the supergroups (2.27) must be $\mathrm{GL}\left(V_{0}\right) \times \mathrm{GL}\left(V_{1}\right)$ and $\mathrm{GL}(V)$, respectively. Their odd dimensions are also easy to deduce from this fact and they are $2 \operatorname{dim} V_{0} \operatorname{dim} V_{1}$ and $(\operatorname{dim} V)^{2}$, respectively. Moreover, these supergroups act on the supermanifold $S V$ according to the morphisms $S \Psi$ and $\Psi_{S}$, respectively.

3. Superlinearity. It has been recognized that, in the supermanifold category we have dealt with in [OASV2], the supermanifold $\mathbf{R}^{1 \mid 1}=\left(\mathbf{R}, C_{\mathbf{R}}^{\infty} \otimes \wedge\left(\mathbf{R}^{*}\right)\right)$ plays the same role as $\mathbf{R}$ does in the category of $C^{\infty}$ manifolds. This is to be understood 
in the sense that, for any coordinate neighborhood $U$ of a given supermanifold $(M, \mathscr{A})$, there is a one-to-one correspondence

$$
\mathscr{A}(U) \leftrightarrow \operatorname{Mor}_{S}\left((U, \mathscr{A} \mid U),\left(\mathbf{R}, C_{\mathbf{R}}^{\infty} \otimes \bigwedge\left(\mathbf{R}^{*}\right)\right)\right) .
$$

Furthermore, this correspondence can be turned into a superalgebra morphism (cf. [OASV2]). This involves the introduction of two supermanifold morphisms

$$
\begin{aligned}
& s: \mathbf{R}^{1 \mid 1} \times \mathbf{R}^{1 \mid 1} \rightarrow \mathbf{R}^{1 \mid 1} \\
& m: \mathbf{R}^{1 \mid 1} \times \mathbf{R}^{1 \mid 1} \rightarrow \mathbf{R}^{1 \mid 1}
\end{aligned}
$$

that endow $\mathbf{R}^{1 \mid 1}$ with the structure of an abstract superalgebra (i.e., associativity, commutativity and distributivity hold true by requiring that certain diagrams involving the morphisms $s$ and $m$ be commutative). Then, any two supermanifold morphisms $\varphi, \psi \in \operatorname{Mor}_{S}\left((U, \mathscr{A} \mid U),\left(\mathbf{R}, C_{\mathbf{R}}^{\infty} \otimes \wedge\left(\mathbf{R}^{*}\right)\right)\right)$ can be summed and multiplied according to

$$
\varphi+\psi:=s \circ(\varphi \times \psi) \text { and } \varphi \cdot \psi:=m \circ(\varphi \times \psi)
$$

respectively. Free $\mathbf{R}^{1 \mid 1}$-modules are then constructed as usual; namely, by just taking a direct product of copies of $\mathbf{R}^{\mathbf{1 | 1}}$ with itself and endowing the resulting supermanifold with sum and scalar multiplication morphisms defined componentwise (cf. [OASV1] and [OASV2]). Thus, if we denote by $\mathbf{R}^{k \mid k}$ the supermanifold $\mathbf{R}^{1 \mid 1} \times \mathbf{R}^{1 \mid 1} \times \cdots \times \mathbf{R}^{1 \mid 1}(k$ copies $)$, then

$$
\sigma_{k}: \mathbf{R}^{k \mid k} \times \mathbf{R}^{k \mid k} \rightarrow \mathbf{R}^{k \mid k}, \quad \mu_{k}: \mathbf{R}^{1 \mid 1} \times \mathbf{R}^{k \mid k} \rightarrow \mathbf{R}^{k \mid k}
$$

are defined in terms of $s$ and $m$ and the projection morphisms

$$
\begin{aligned}
\tau_{j}: \mathbf{R}^{k \mid k} \times \mathbf{R}^{k \mid k} \rightarrow \mathbf{R}^{1 \mid 1} ; & \text { projection onto the } j \text { th factor, } 1 \leq j \leq 2 k, \\
\rho_{i}: \mathbf{R}^{1 \mid 1} \times \mathbf{R}^{k \mid k} \rightarrow \mathbf{R}^{1 \mid 1} ; & \text { projection onto the }(i+1) \text { st factor, } 0 \leq i \leq k,
\end{aligned}
$$

as follows:

$$
\begin{aligned}
& \sigma_{k}=s \circ\left(\tau_{1} \times \tau_{k+1}\right) \times s \circ\left(\tau_{2} \times \tau_{k+2}\right) \times \cdots \times s \circ\left(\tau_{k} \times \tau_{2 k}\right), \\
& \mu_{k}=m \circ\left(\rho_{0} \times \rho_{1}\right) \times m \circ\left(\rho_{0} \times \rho_{2}\right) \times \cdots \times m \circ\left(\rho_{0} \times \rho_{k}\right) .
\end{aligned}
$$

In other words, if we denote by

$$
p_{i}: \mathbf{R}^{k \mid k} \rightarrow \mathbf{R}^{1 \mid 1}, \quad 1 \leq i \leq k,
$$

the projection morphism onto the $i$ th factor, we will have

$$
p_{i} \circ \sigma_{k}=s \circ\left(\tau_{i} \times \tau_{k+i}\right) \text { and } p_{i} \circ \mu_{k}=m \circ\left(\rho_{0} \times \rho_{i}\right),
$$

which completely determine the morphisms $\sigma_{k}$ and $\mu_{k}$.

Having this structure introduced in the supermanifold $\mathbf{R}^{k \mid k}$, it makes sense to ask whether or not a given supermanifold morphism

$$
L: \mathbf{R}^{k \mid k} \rightarrow \mathbf{R}^{1 \mid 1}
$$

preserves it that is, whether or not $L$ satisfies the equalities

$$
\begin{gathered}
L \circ \sigma_{k}=s \circ\left\{L \circ\left(\tau_{1} \times \tau_{2} \times \cdots \times \tau_{k}\right) \times L \circ\left(\tau_{k+1} \times \tau_{k+1} \times \cdots \times \tau_{2 k}\right)\right\}, \\
L \circ \mu_{k}=m \circ\left\{\rho_{0} \times L \circ\left(\rho_{1} \times \rho_{2} \times \cdots \times \rho_{k}\right)\right\},
\end{gathered}
$$


understood as supermanifold morphisms. We have called such an $L$ superlinear (cf. [OASV2]). It is our purpose here to characterize all superlinear supermanifold morphisms $L: \mathbf{R}^{k \mid k} \rightarrow \mathbf{R}^{1 \mid 1}$.

We shall proceed as in $\S 1$ by noting, first of all, that $L$ is uniquely determined by the superalgebra morphism (cf. [Kostant] or [Leĭtes])

$$
L^{\#}: C^{\infty}(\mathbf{R}) \otimes \bigwedge\left(\mathbf{R}^{*}\right) \rightarrow C^{\infty}\left(\mathbf{R}^{k}\right) \otimes \bigwedge\left(\left(\mathbf{R}^{k}\right)^{*}\right) .
$$

This is in turn completely determined by the pair of sections

$$
L^{\#} t \in\left\{C^{\infty}\left(\mathbf{R}^{k}\right) \otimes \bigwedge\left(\left(\mathbf{R}^{k}\right)^{*}\right)\right\}_{0} \quad \text { and } \quad L^{\#} \zeta \in\left\{C^{\infty}\left(\mathbf{R}^{k}\right) \otimes \bigwedge\left(\left(\mathbf{R}^{k}\right)^{*}\right)\right\}_{1}
$$

whenever $\{t, \zeta\}$ is a coordinate system on $\mathbf{R}^{1 \mid 1}$ (cf. [Lertes]). Moreover, the definition of $\mathbf{R}^{k \mid k}$ as the product $\mathbf{R}^{\mathbf{1} \mid \mathbf{1}} \times \mathbf{R}^{\mathbf{1} \mid 1} \times \cdots \times \mathbf{R}^{1 \mid 1}$ implies that, for any coordinate system $\{t, \varsigma\}$ on $\mathbf{R}^{1 \mid 1},\left\{p_{i}^{\#} t, p_{i}^{\#} \varsigma ; 1 \leq i \leq k\right\}$ is a coordinate system on $\mathbf{R}^{k \mid k}$ (cf. [Leĭtes]). This means that we can write $p_{i}^{\#} t$ in the form

$$
p_{i}^{\#} t=t_{i}+z_{i}, \quad 1 \leq i \leq k
$$

with $t_{i} \in C^{\infty}\left(\mathbf{R}^{k}\right),\left(t_{1}, t_{2}, \ldots, t_{k}\right)$ a coordinate system on $\mathbf{R}^{k}$, and $z_{i} \in \mathscr{J}^{2}\left(\mathbf{R}^{k \mid k}\right)$ which, by definition, is the square of the ideal $\mathscr{J}\left(\mathbf{R}^{k \mid k}\right)$ generated by the odd subspace $\left\{C^{\infty}\left(\mathbf{R}^{k}\right) \otimes \bigwedge\left(\left(\mathbf{R}^{k}\right)^{*}\right)\right\}_{1}$. Evidently, $z_{i}$ is nilpotent and, in fact, $z_{i}^{[k / 2]+1}=$ 0 .

In a similar fashion, we shall write the even coordinates $\left\{\tau_{j}^{\#} t ; 1 \leq j \leq 2 k\right\}$ and $\left\{\rho_{i}^{\#} t ; 0 \leq i \leq k\right\}$ of $\mathbf{R}^{2 k \mid 2 k}$ and $\mathbf{R}^{k+1 \mid k+1}$ respectively in the form

$$
\begin{aligned}
& \tau_{j}^{\#} t=T_{j}+\eta_{j}, \quad T_{j} \in C^{\infty}\left(\mathbf{R}^{2 k}\right), \eta_{j} \in \mathcal{J}^{2}\left(\mathbf{R}^{2 k \mid 2 k}\right), 1 \leq j \leq 2 k, \\
& \rho_{i}^{\#} t=\theta_{i}+\kappa_{i}, \quad \theta_{i} \in C^{\infty}\left(\mathbf{R}^{k+1}\right), \kappa_{i} \in \mathcal{J}^{2}\left(\mathbf{R}^{k+1 \mid k+1}\right), 0 \leq i \leq k,
\end{aligned}
$$

so that $\left(T_{1}, T_{2}, \ldots, T_{2 k}\right)$ and $\left(\theta_{0}, \theta_{1}, \ldots, \theta_{k}\right)$ are coordinate systems on $\mathbf{R}^{2 k}$ and $\mathbf{R}^{k+1}$, respectively.

We shall now state the following partial results.

3.1 LEMMA. Let the notation be as above. Then, for any $C^{\infty}$ function $f$ on $\mathbf{R}^{k}$, we have

(i) $\sigma_{k}^{\#} f \equiv f \circ \tilde{\sigma}_{k}+\sum_{i=1}\left[\eta_{i}+\eta_{k+i}-\sigma_{k}^{\#} z_{i}\right]\left(\partial_{i} f\right) \circ \tilde{\sigma}_{k} \operatorname{Mod} \mathcal{J}^{4}\left(\mathbf{R}^{2 k \mid 2 k}\right)$,

(ii) $\mu_{k}^{\#} f \equiv f \circ \tilde{\mu}_{k}+\sum_{i=1}\left[\theta_{0} \kappa_{i}+\kappa_{0} \theta_{i}+\rho_{0}^{\#} \varsigma \rho_{i}^{\#} \varsigma-\mu_{k}^{\#} z_{i}\right]\left(\partial_{i} f\right) \circ \tilde{\mu}_{k}$

$\operatorname{Mod} \mathscr{J}^{4}\left(\mathbf{R}^{k+1 \mid k+1}\right)$,

(iii) $\left(\tau_{1} \times \tau_{2} \times \cdots \times \tau_{k}\right)^{\#} f \equiv f \circ \tilde{\pi}_{1}+\sum_{i=1} \eta_{i}\left(\partial_{i} f\right) \circ \tilde{\pi}_{1} \operatorname{Mod} \mathcal{J}^{4}\left(\mathbf{R}^{2 k \mid 2 k}\right)$,

(iv) $\left(\tau_{k+1} \times \tau_{k+2} \times \cdots \times \tau_{2 k}\right)^{\#} f \equiv f \circ \tilde{\pi}_{2}+\sum_{i=1} \eta_{k+i}\left(\partial_{i} f\right) \circ \tilde{\pi}_{2}$

$\operatorname{Mod} \mathscr{J}^{4}\left(\mathbf{R}^{2 k \mid 2 k}\right)$

(v) $\left(\rho_{1} \times \rho_{2} \times \cdots \times \rho_{k}\right)^{\#} f \equiv f \circ \tilde{\pi}_{0}+\sum_{i=1} \kappa_{i}\left(\partial_{i} f\right) \circ \tilde{\pi}_{0} \operatorname{Mod} \mathscr{J}^{4}\left(\mathbf{R}^{k+1 \mid k+1}\right)$, 
where we have put

$$
\begin{aligned}
\tilde{\sigma}_{k}\left(T_{1}, T_{2}, \ldots, T_{2 k}\right) & =\left(T_{1}+T_{k+1}, T_{2}+T_{k+2}, \ldots, T_{k}+T_{2 k}\right), \\
\tilde{\pi}_{1}\left(T_{1}, T_{2}, \ldots, T_{2 k}\right) & =\left(T_{1}, T_{2}, \ldots, T_{k}\right), \\
\tilde{\pi}_{2}\left(T_{1}, T_{2}, \ldots, T_{2 k}\right) & =\left(T_{k+1}, T_{k+2}, \ldots, T_{2 k}\right), \\
\tilde{\mu}_{k}\left(\theta_{0}, \theta_{1}, \ldots, \theta_{k}\right) & =\left(\theta_{0} \theta_{1}, \theta_{0} \theta_{2}, \ldots, \theta_{0} \theta_{k}\right), \\
\tilde{\pi}_{0}\left(\theta_{0}, \theta_{1}, \ldots, \theta_{k}\right) & =\left(\theta_{1}, \theta_{2}, \ldots, \theta_{k}\right) .
\end{aligned}
$$

PROOF. It has already been explained in [Kostant] and [Lertes] how to prove statements like these. The idea consists of writing $f \in C^{\infty}\left(\mathbf{R}^{k}\right)$ as its formal Taylor expansion,

$$
f=\sum_{\nu \in M_{k}} \frac{1}{\nu !} \frac{\partial^{\nu} f}{\partial t^{\nu}}(0) t^{\nu}
$$

where $M_{k}=\left\{\nu=\left(\nu_{1}, \nu_{2}, \ldots, \nu_{k}\right) \mid \nu_{i} \in \mathbf{N} \cup\{0\}\right\}$, and for any sequence $\nu=$ $\left(\nu_{1}, \nu_{2}, \ldots, \nu_{k}\right) \in M_{k}, \nu !=\nu_{1} ! \nu_{2} ! \cdots \nu_{k} !, t^{\nu}=t_{1}^{\nu_{1}} t_{2}^{\nu_{2}} \cdots t_{k}^{\nu_{k}}$, and

$$
\frac{\partial^{\nu} f}{\partial t^{\nu}}=\frac{\partial^{\nu_{1}+\nu_{2}+\cdots+\nu_{k} f}}{\partial t_{1}^{\nu_{1}} \partial t_{2}^{\nu_{2}} \cdots \partial t_{k}^{\nu_{k}}} .
$$

The effect of any superalgebra morphism, $\Phi^{\#}$ say, on the $C^{\infty}$ function $f$ can be computed via

$$
\Phi^{\#} f=\sum_{\nu \in M_{k}} \frac{1}{\nu !} \frac{\partial^{\nu} f}{\partial t^{\nu}}(0)\left(\Phi^{\#} t\right)^{\nu}
$$

which makes sense because $\Phi^{\#} t$ can be written in the form $\tilde{\Phi} t+N$, where $N$ is nilpotent, and hence $\Phi^{\#} f$ is expressed as $f \circ \tilde{\Phi}$ plus a finite number of terms involving all the nilpotency and the partial derivatives of $f$ (composed with $\tilde{\Phi}$ ). Thus, for example, in proving (i), we note that

$$
\begin{aligned}
\sigma_{k}^{\#} t_{i} & =\sigma_{k}^{\#} p_{i}^{\#} t-\sigma_{k}^{\#} z_{i}=\tau_{i}^{\#} t+\tau_{k+i}^{\#} t-\sigma_{k}^{\#} z_{i} \\
& =T_{i}+T_{k+i}+\left(\eta_{i}+\eta_{k+i}-\sigma_{k}^{\#} z_{i}\right) \equiv T_{i}+T_{k+i} \quad \operatorname{Mod} \mathcal{J}^{2}\left(\mathbf{R}^{2 k \mid 2 k}\right) .
\end{aligned}
$$

Since for any $i$ and $j$ we have

$$
\left(\eta_{i}+\eta_{k+i}-\sigma_{k}^{\#} z_{i}\right)\left(\eta_{j}+\eta_{k+j}-\sigma_{k}^{\#} z_{j}\right) \in \operatorname{Mod} \mathscr{J}^{4}\left(\mathbf{R}^{2 k \mid 2 k}\right)
$$

it is easy to see that (i) holds. The other statements are proved similarly.

Having these results in mind, we can start our characterization of the superlinear supermanifold morphisms $L: \mathbf{R}^{k \mid k} \rightarrow \mathbf{R}^{1 \mid 1}$ by writing $L^{\#} t$ and $L^{\#} \zeta$ in the form

$$
\begin{aligned}
L^{\#} t & =L_{0}+\sum_{1 \leq i<j \leq k} L_{i j} p_{i}^{\#} \varsigma p_{j}^{\#} \varsigma+\mathcal{J}^{4}\left(\mathbf{R}^{k \mid k}\right), \\
L^{\#} \varsigma & =\sum_{1 \leq i \leq k} L_{i} p_{i}^{\#} \varsigma+\sum_{1 \leq h<i<j \leq k} L_{h i j} p_{h}^{\#} \varsigma p_{i}^{\#} \varsigma p_{j}^{\#} \varsigma+\mathcal{J}^{5}\left(\mathbf{R}^{k \mid k}\right)
\end{aligned}
$$

respectively, with $L_{0}, L_{i}, L_{i j}$, and $L_{h i j}$ in $C^{\infty}\left(\mathbf{R}^{k}\right)$. Then, we note that $L$ is superlinear if and only if for any coordinate system $\{t, \zeta\}$ on $\mathbf{R}^{1 \mid 1}$, the following equations are satisfied (cf. [OASV2]):

(a)

$$
\sigma_{k}^{\#}\left(L^{\#} t\right)=\left(\tau_{1} \times \tau_{2} \times \cdots \times \tau_{k}\right)^{\#}\left(L^{\#} t\right)+\left(\tau_{k+1} \times \tau_{k+2} \times \cdots \times \tau_{2 k}\right)^{\#}\left(L^{\#} t\right)
$$


(b)

$$
\sigma_{k}^{\#}\left(L^{\#} \zeta\right)=\left(\tau_{1} \times \tau_{2} \times \cdots \times \tau_{k}\right)^{\#}\left(L^{\#} \zeta\right)+\left(\tau_{k+1} \times \tau_{k+2} \times \cdots \times \tau_{2 k}\right)^{\#}\left(L^{\#} \zeta\right),
$$

(c)

$$
\mu_{k}^{\#}\left(L^{\#} t\right)=\left(\rho_{0}^{\#} t\right)\left(\rho_{1} \times \cdots \times \rho_{k}\right)^{\#}\left(L^{\#} t\right)+\left(\rho_{0}^{\#} \varsigma\right)\left(\rho_{1} \times \cdots \times \rho_{k}\right)^{\#}\left(L^{\#} \varsigma\right),
$$

(d)

$$
\mu_{k}^{\#}\left(L^{\#} \varsigma\right)=\left(\rho_{0}^{\#} t\right)\left(\rho_{1} \times \cdots \times \rho_{k}\right)^{\#}\left(L^{\#} \zeta\right)+\left(\rho_{0}^{\#} \zeta\right)\left(\rho_{1} \times \cdots \times \rho_{k}\right)^{\#}\left(L^{\#} t\right) .
$$

Thus, our second partial result reads as follows.

3.2 LemMA. Let the notation be as above. As a consequence of (a), we have

$$
L^{\#} t=L_{0} \quad \text { where } L_{0}\left(t_{1}, t_{2}, \ldots, t_{k}\right)=\sum_{1 \leq i \leq k} A_{i} t_{i}, A_{i} \in \mathbf{R} .
$$

Furthermore, as a consequence of the actual proof of this lemma one is led to the following.

3.3 COROllary. Let $\mathbf{R}^{k \mid k}$ be the supermanifold $\mathbf{R}^{1 \mid 1} \times \mathbf{R}^{1 \mid 1} \times \cdots \times \mathbf{R}^{1 \mid 1}$ and let

$$
p_{i}: \mathbf{R}^{k \mid k} \rightarrow \mathbf{R}^{1 \mid 1} \quad(1 \leq i \leq k)
$$

be the projection morphism onto the ith factor. Then, for any $C^{\infty}$ function $f$ on $\mathbf{R}$, we have $p_{i}^{\#} f=f \circ \tilde{p}_{i}$, where $\tilde{p}_{i}: \mathbf{R}^{k} \rightarrow \mathbf{R}$ denotes the ith projection morphism in the $C^{\infty}$ category.

Therefore, if we combine this with the results of Lemma 3.1, we get

3.4 Proposition. For any $C^{\infty}$ function $f$ on $\mathbf{R}^{k}$, we have

(i) $\sigma_{k}^{\#} f=f \circ \tilde{\sigma}_{k}$,

(ii) $\mu_{k}^{\#} f=f \circ \tilde{\mu}_{k}+\sum_{1 \leq i \leq k}\left(\partial_{i} f\right) \circ \tilde{\mu}_{k} \rho_{0}^{\#} \varsigma \rho_{i}^{\#} \varsigma$,

(iii) $\left(\tau_{1} \times \tau_{2} \times \cdots \times \tau_{k}\right){ }^{\#} f=f \circ \tilde{\pi}_{1}$,

(iv) $\left(\tau_{k+1} \times \tau_{k+2} \times \cdots \times \tau_{2 k}\right) \# f=f \circ \tilde{\pi}_{2}$,

(v) $\left(\rho_{1} \times \rho_{2} \times \cdots \times \rho_{k}\right)^{\#} f=f \circ \tilde{\pi}_{0}$ where $\tilde{\sigma}_{k}, \tilde{\mu}_{k}, \tilde{\pi}_{0}, \tilde{\pi}_{1}$, and $\tilde{\pi}_{2}$ are as in Lemma 3.1.

PROOFS OF 3.2 AND 3.3. This is a straightforward computation based on the results of Lemma 3.1; first of all, using (3.15), the left-hand side of (a) gives

$$
\begin{aligned}
\sigma_{k}^{\#}\left(L^{\#} t\right)= & \sigma_{k}^{\#}\left(L_{0}+\sum_{1 \leq i<j \leq k} L_{i j} p_{i}^{\#} \varsigma p_{j}^{\#} \varsigma+\mathcal{J}^{4}\left(\mathbf{R}^{k \mid k}\right)\right) \\
= & L_{0} \circ \tilde{\sigma}_{k}+\sum_{1 \leq i \leq k}\left[\eta_{i}+\eta_{k+i}-\sigma_{k}^{\#} z_{i}\right]\left(\partial_{i} L_{0}\right) \circ \tilde{\sigma}_{k} \\
& +\sum_{1 \leq i<j \leq k} L_{i j} \circ \tilde{\sigma}_{k}\left(\tau_{i}^{\#} \varsigma+\tau_{k+i}^{\#} \varsigma\right)\left(\tau_{j}^{\#} \varsigma+\tau_{k+j}^{\#} \varsigma\right)+\mathcal{J}^{4}\left(\mathbf{R}^{2 k \mid 2 k}\right) .
\end{aligned}
$$


On the other hand, the right-hand side of (a) yields

$$
\begin{aligned}
\left(\tau_{1} \times \tau_{2} \times \cdots \times \tau_{k}\right)^{\#}\left(L^{\#} t\right)+\left(\tau_{k+1} \times \tau_{k+2} \times \cdots \times \tau_{2 k}\right)^{\#}\left(L^{\#} t\right) \\
=\left(\tau_{1} \times \tau_{2} \times \cdots \times \tau_{k}\right)^{\#}\left(L_{0}+\sum_{1 \leq i<j \leq k} L_{i j} p_{i}^{\#} \varsigma p_{j}^{\#} \varsigma+\mathcal{J}^{4}\left(\mathbf{R}^{k \mid k}\right)\right) \\
\quad+\left(\tau_{k+1} \times \tau_{k+2} \times \cdots \times \tau_{2 k}\right)^{\#}\left(L_{0}+\sum_{1 \leq i<j \leq k} L_{i j} p_{i}^{\#} \varsigma p_{j}^{\#} \varsigma+\mathcal{J}^{4}\left(\mathbf{R}^{k \mid k}\right)\right) \\
=L_{0} \circ \tilde{\pi}_{1}+\sum_{1 \leq i \leq k} \eta_{i}\left(\partial_{i} L_{0}\right) \circ \tilde{\pi}_{1}+\sum_{1 \leq i<j \leq k} L_{i j} \circ \tilde{\pi}_{1} \tau_{i}^{\#} \varsigma \tau_{j}^{\#} \varsigma \\
\quad+L_{0} \circ \tilde{\pi}_{2}+\sum_{1 \leq i \leq k} \eta_{k+i}\left(\partial_{i} L_{0}\right) \circ \tilde{\pi}_{2} \\
\quad+\sum_{1 \leq i<j \leq k} L_{i j} \circ \tilde{\pi}_{2} \tau_{k+i}^{\#} \varsigma \tau_{k+i}^{\#} \varsigma+\mathcal{J}^{4}\left(\mathbf{R}^{2 k \mid 2 k}\right) .
\end{aligned}
$$

Equality of these two implies, for the zeroth-order terms, that

$$
L_{0}\left(T_{1}+T_{k+1}, \ldots, T_{k}+T_{2 k}\right)=L_{0}\left(T_{1}, \ldots, T_{k}\right)+L_{0}\left(T_{k+1}, \ldots, T_{2 k}\right) .
$$

Since $L_{0}$ is $C^{\infty}$ we can differentiate both sides of this equation with respect to $T_{i}$ and $T_{k+i}(1 \leq i \leq k)$ and get

$$
\left(\partial_{i} L_{0}\right)\left(T_{1}, \ldots, T_{k}\right)=\left(\partial_{i} L_{0}\right)\left(T_{1}+T_{k+1}, \ldots, T_{k}+T_{2 k}\right)=\left(\partial_{i} L_{0}\right)\left(T_{k+1}, \ldots, T_{2 k}\right) .
$$

That is, $\partial_{i} L_{0}=$ constant $=A_{i}$ and, in view of $(*)$, it follows that

$$
L_{0}\left(t_{1}, t_{2}, \ldots, t_{k}\right)=\sum_{1 \leq i \leq k} A_{i} t_{i}, \quad A_{i} \in \mathbf{R} .
$$

Using this fact, we can now compare the second-order terms of the equality (a) and find that

$$
\begin{aligned}
& \sum_{1 \leq i<j \leq k} L_{i j} \circ \tilde{\sigma}_{k}\left(\tau_{i}^{\#} \varsigma+\tau_{k+i}^{\#} \varsigma\right)\left(\tau_{j}^{\#} \varsigma+\tau_{k+j}^{\#} \varsigma\right)-\sum_{1 \leq h \leq k} A_{h} \sigma_{k}^{\#} z_{h} \\
& \left.\quad \equiv \sum_{1 \leq i<j \leq k}\left\{\left(L_{i j} \circ \tilde{\pi}_{1}\right) \tau_{i}^{\#} \varsigma \tau_{j}^{\#} \varsigma+\left(L_{i j} \circ \tilde{\pi}_{2}\right) \tau_{k+i}^{\#} \varsigma \tau_{k+j}^{\#} \varsigma\right)\right\} \quad \operatorname{Mod} \mathcal{J}^{4}\left(\mathbf{R}^{2 k \mid 2 k}\right) .
\end{aligned}
$$

If we now assume that

$$
z_{h}=\sum_{1 \leq i<j \leq k} Z_{i j}^{h} p_{i}^{\#} \varsigma p_{j}^{\#} \varsigma+\mathcal{J}^{4}\left(\mathbf{R}^{k \mid k}\right), \quad Z_{i j}^{h} \in C^{\infty}\left(\mathbf{R}^{k}\right)
$$

then

$$
\sigma_{k}^{\#} z_{h} \equiv \sum_{1 \leq i<j \leq k} Z_{i j}^{h} \circ \tilde{\sigma}_{k}\left(\tau_{i}^{\#} \varsigma+\tau_{k+i}^{\#} \varsigma\right)\left(\tau_{j}^{\#} \varsigma+\tau_{k+j}^{\#} \varsigma\right) \quad \operatorname{Mod} \mathscr{J}^{4}\left(\mathbf{R}^{2 k \mid 2 k}\right) .
$$

Therefore, we conclude that

$$
\begin{aligned}
L_{i j}\left(T_{1}+\right. & \left.T_{k+1}, \ldots, T_{k}+T_{2 k}\right)-\sum_{h} A_{h} Z_{i j}^{h}\left(T_{1}+T_{k+1}, \ldots, T_{k}+T_{2 k}\right) \\
& =L_{i j}\left(T_{1}, \ldots, T_{k}\right)
\end{aligned}
$$




$$
\begin{gathered}
L_{i j}\left(T_{1}+T_{k+1}, \ldots, T_{k}+T_{2 k}\right)-\sum_{h} A_{h} Z_{i j}^{h}\left(T_{1}+T_{k+1}, \ldots, T_{k}+T_{2 k}\right)=0 \\
L_{i j}\left(T_{1}+T_{k+1}, \ldots, T_{k}+T_{2 k}\right)-\sum_{h} A_{h} Z_{i j}^{h}\left(T_{1}+T_{k+1}, \ldots, T_{k}+T_{2 k}\right) \\
=L_{i j}\left(T_{k+1}, \ldots, T_{2 k}\right) .
\end{gathered}
$$

It is clear from the right-hand sides of this set of equations that

$$
L_{i j}\left(T_{1}, \ldots, T_{k}\right)=0=L_{i j}\left(T_{k+1}, \ldots, T_{2 k}\right)
$$

and hence, using this information on the left-hand sides, it follows that

$$
\sum_{h} A_{h} Z_{i j}^{h}\left(T_{1}, \ldots, T_{k}\right)=0
$$

Since the $A_{h}$ 's are arbitrary, we get

$$
Z_{i j}^{h}\left(T_{1}, \ldots, T_{k}\right)=0
$$

In particular, $z_{h} \in \mathscr{J}^{4}\left(\mathbf{R}^{k \mid k}\right)$.

The rest of the proof can be handled by induction on $s$, where it is assumed that $z_{h} \in \mathscr{J}^{2 s}\left(\mathbf{R}^{k \mid k}\right)$ and that

$$
L^{\#} t=L_{0}+\sum_{1 \leq i_{1}<i_{2}<\cdots<i_{2} \leq k} L_{i_{1} i_{2} \cdots i_{2} s} p_{i_{1}}^{\#} \varsigma p_{i_{2}}^{\#} \varsigma \cdots p_{i_{2} s}^{\#} \varsigma+\mathcal{J}^{2(s+1)}\left(\mathbf{R}^{k \mid k}\right) .
$$

One then proves that $L_{i_{1} i_{2} \cdots i_{2 s}}=0$ and that $z_{h}$ belongs to $\mathcal{J}^{2(s+1)}\left(\mathbf{R}^{k \mid k}\right)$. Since this process has to stop as soon as $2 s>k$, the proof is complete.

The second step towards our characterization of superlinearity consists of looking at the equation

(b) $\sigma_{k}^{\#}\left(L^{\#} \varsigma\right)=\left(\tau_{1} \times \tau_{2} \times \cdots \times \tau_{k}\right)^{\#}\left(L^{\#} \varsigma\right)+\left(\tau_{k+1} \times \tau_{k+2} \times \cdots \times \tau_{2 k}\right)^{\#}\left(L^{\#} \varsigma\right)$

with $L^{\#} \varsigma$ written as in (3.15) and conclude the following.

3.5 LEMMA. Let the notation be as above. As a consequence of (b), we have

$$
L^{\#} \varsigma=\sum_{1 \leq i \leq k} B_{i} p_{i}^{\#} \varsigma ; \quad B_{i} \in \mathbf{R}
$$

ProOF. The idea is the same as before. We write

$$
L^{\#} \varsigma=\sum_{1 \leq i \leq k} L_{i} p_{i}^{\#} \varsigma+\sum_{1 \leq h<i<j \leq k} L_{h i j} p_{h}^{\#} \varsigma p_{i}^{\#} \varsigma p_{j}^{\#} \varsigma+\mathcal{J}^{5}\left(\mathbf{R}^{k \mid k}\right)
$$


Then, the left-hand side of (b) gives

$$
\begin{aligned}
\sigma_{k}^{\#}\left(L^{\#} \varsigma\right)= & \sigma_{k}^{\#}\left(\sum_{1 \leq i \leq k} L_{i} p_{i}^{\#} \varsigma+\sum_{1 \leq h<i<j \leq k} L_{h i j} p_{h}^{\#} \varsigma p_{i}^{\#} \varsigma p_{j}^{\#} \varsigma+\mathcal{J}^{5}\left(\mathbf{R}^{k \mid k}\right)\right) \\
= & \sum_{1 \leq i \leq k}\left(L_{i} \circ \tilde{\sigma}_{k}\right) \sigma_{k}^{\#} p_{i}^{\#} \varsigma \\
& +\sum_{1 \leq h<i<j \leq k}\left(L_{h i j} \circ \tilde{\sigma}_{k}\right) \sigma_{k}^{\#} p_{h}^{\#} \varsigma \sigma_{k}^{\#} p_{i}^{\#} \varsigma \sigma_{k}^{\#} p_{j}^{\#} \varsigma+\mathcal{J}^{5}\left(\mathbf{R}^{2 k \mid 2 k}\right) \\
= & \sum_{1 \leq i \leq k}\left(L_{i} \circ \tilde{\sigma}_{k}\right)\left(\tau_{i}^{\#} \varsigma+\tau_{k+i}^{\#} \varsigma\right) \\
& +\sum_{1 \leq h<i<j \leq k}\left(L_{h i j} \circ \tilde{\sigma}_{k}\right)\left(\tau_{h}^{\#} \varsigma+\tau_{k+h}^{\#} \varsigma\right)\left(\tau_{i}^{\#} \varsigma+\tau_{k+i}^{\#} \varsigma\right)\left(\tau_{j}^{\#} \varsigma+\tau_{k+j}^{\#} \varsigma\right) \\
& +\mathcal{J}^{5}\left(\mathbf{R}^{2 k \mid 2 k}\right)
\end{aligned}
$$

where use has been made of 3.3 and 3.4. On the other hand, the right-hand side of (b) yields

$$
\begin{aligned}
&\left(\tau_{1} \times \tau_{2} \times \cdots \times \tau_{k}\right)^{\#}\left(L^{\#} \varsigma\right)+\left(\tau_{k+1} \times \tau_{k+2} \times \cdots \times \tau_{2 k}\right)^{\#}\left(L^{\#} \varsigma\right) \\
&=\left(\tau_{1} \times \cdots \times \tau_{k}\right)^{\#}\left(\sum_{1 \leq i \leq k} L_{i} p_{i}^{\#} \varsigma\right)+\left(\tau_{k+1} \times \cdots \times \tau_{2 k}\right)^{\#}\left(\sum_{1 \leq i \leq k} L_{i} p_{i}^{\#} \varsigma\right) \\
&+\left(\tau_{1} \times \cdots \times \tau_{k}\right)^{\#}\left(\sum_{1 \leq h<i<j \leq k} L_{h i j} p_{h}^{\#} \varsigma p_{i}^{\#} \varsigma p_{j}^{\#} \varsigma+\mathcal{J}^{5}\left(\mathbf{R}^{k \mid k}\right)\right) \\
&+\left(\tau_{k+1} \times \cdots \times \tau_{2 k}\right)^{\#}\left(\sum_{1 \leq h<i<j \leq k} L_{h i j} p_{h}^{\#} \varsigma p_{i}^{\#} \varsigma p_{j}^{\#} \varsigma+\mathcal{J}^{5}\left(\mathbf{R}^{k \mid k}\right)\right) \\
&= \sum_{1 \leq i \leq k}\left(L_{i} \circ \tilde{\pi}_{1}\right) \tau_{i}^{\#} \varsigma+\sum_{1 \leq i \leq k}\left(L_{i} \circ \tilde{\pi}_{2}\right) \tau_{k+i}^{\#} \varsigma \\
&+\sum_{1 \leq h<i<j \leq k}\left\{\left(L_{h i j} \circ \tilde{\pi}_{1}\right) \tau_{h}^{\#} \varsigma \tau_{i}^{\#} \varsigma \tau_{j}^{\#} \varsigma+\left(L_{h i j} \circ \tilde{\pi}_{2}\right) \tau_{k+h}^{\#} \varsigma \tau_{k+i}^{\#} \varsigma \tau_{k+j}^{\#} \varsigma\right\} \\
&+\mathcal{J}^{5}\left(\mathbf{R}^{2 k \mid 2 k}\right) .
\end{aligned}
$$

Equality of these two implies, for the first-order terms, that

$$
L_{i} \circ \tilde{\pi}_{1}=L_{i} \circ \tilde{\sigma}_{k}=L_{i} \circ \tilde{\pi}_{2} .
$$

In other words, for all $\left(T_{1}, T_{2}, \ldots, T_{2 k}\right) \in \mathbf{R}^{2 k}$

$$
L_{i}\left(T_{1}, \ldots, T_{k}\right)=L_{i}\left(T_{1}+T_{k+1}, \ldots, T_{k}+T_{2 k}\right)=L_{i}\left(T_{k+1}, \ldots, T_{2 k}\right)
$$

which immediately implies that

$$
L_{i}=\text { constant }=B_{i} .
$$

Now, comparison of the third-order terms shows that

$$
L_{h i j} \circ \tilde{\pi}_{1}=L_{h i j} \circ \tilde{\sigma}_{k}=L_{h i j} \circ \tilde{\pi}_{2}=0
$$


and proceeding inductively one easily proves that, in fact, there will be no higherorder terms in the coordinate expression of $L^{\#} \zeta$.

The third and last step consists of making sure that (c) and (d) independently imply that the constants $A_{i}$ and $B_{i}$ of 3.2 and 3.5 have to be equal for $L$ to be superlinear. That is,

3.6 PROPOSITION. A supermanifold morphism $L: \mathbf{R}^{k \mid k} \rightarrow \mathbf{R}^{1 \mid 1}$ is superlinear if and only if its coordinate expression is of the form

$$
L^{\#} t=\sum_{1 \leq i \leq k} A_{i} p_{i}^{\#} t \quad \text { and } \quad L^{\#} \varsigma=\sum_{1 \leq i \leq k} A_{i} p_{i}^{\#} \varsigma, \quad A_{i} \in \mathbf{R}
$$

where $\{t, \varsigma\}$ is any coordinate system of $\mathbf{R}^{1 \mid 1}$.

PROOF. Let us write $L^{\#} t=\sum_{1 \leq i \leq k} A_{i} p_{i}^{\#} t$ and $L^{\#} \varsigma=\sum_{1 \leq i \leq k} B_{i} p_{i}^{\#} \varsigma$ and let us see what the equality

(c) $\mu_{k}^{\#}\left(L^{\#} t\right)=\left(\rho_{0}^{\#} t\right)\left(\rho_{1} \times \cdots \times \rho_{k}\right)^{\#}\left(L^{\#} t\right)+\left(\rho_{0}^{\#} \varsigma\right)\left(\rho_{1} \times \cdots \times \rho_{k}\right)^{\#}\left(L^{\#} \varsigma\right)$

implies. Using 3.4(ii) we get, for the left-hand side of (c),

$$
\begin{aligned}
\mu_{k}^{\#}\left(L^{\#} t\right) & =\mu_{k}^{\#}\left(\sum_{1 \leq i \leq k} A_{i} p_{i}^{\#} t\right)=\sum_{1 \leq i \leq k} A_{i} \mu_{k}^{\#} p_{i}^{\#} t \\
& =\sum_{1 \leq i \leq k} A_{i}\left\{\left(\rho_{0}^{\#} t\right)\left(\rho_{i}^{\#} t\right)+\left(\rho_{0}^{\#} \varsigma\right)\left(\rho_{i}^{\#} \varsigma\right)\right\},
\end{aligned}
$$

while, using 3.4(v), the right-hand of (c) gives

$$
\begin{aligned}
\left(\rho_{0}^{\#} t\right)\left(\rho_{1} \times \cdots \times \rho_{k}\right)^{\#}\left(L^{\#} t\right)+\left(\rho_{0}^{\#} \varsigma\right)\left(\rho_{1} \times \cdots \times \rho_{k}\right)^{\#}\left(L^{\#} \varsigma\right) \\
=\left(\rho_{0}^{\#} t\right)\left(\rho_{1} \times \cdots \times \rho_{k}\right)^{\#}\left(\sum_{1 \leq i \leq k} A_{i} p_{i}^{\#} t\right) \\
+\left(\rho_{0}^{\#} \varsigma\right)\left(\rho_{1} \times \cdots \times \rho_{k}\right)^{\#}\left(\sum_{1 \leq i \leq k} B_{i} p_{i}^{\#} \varsigma\right) \\
=\left(\rho_{0}^{\#} t\right)\left(\sum_{1 \leq i \leq k} A_{i} \rho_{i}^{\#} t\right)+\left(\rho_{0}^{\#} \varsigma\right)\left(\sum_{1 \leq i \leq k} B_{i} \rho_{i}^{\#} \varsigma\right) .
\end{aligned}
$$

Therefore, (c) implies $A_{i}=B_{i}$. It is equally easy to see that (d) leads to the same conclusion.

More generally, in order to consider superlinear morphisms from $\mathbf{R}^{k \mid k}$ into $\mathbf{R}^{m \mid m}$ we use the fact that any supermanifold morphism $\Phi:\left(\mathbf{R}^{k}, \mathscr{R}^{k \mid k}\right) \rightarrow\left(\mathbf{R}^{m}, \mathscr{R}^{m \mid m}\right)$ is completely determined by the $m$-tuple of morphisms $\left(\Phi_{1}, \Phi_{2}, \ldots, \Phi_{m}\right)$, where

$$
\Phi_{j}:=\pi_{j} \circ \Phi:\left(\mathbf{R}^{k}, \mathscr{R}^{k \mid k}\right) \rightarrow\left(\mathbf{R}, \mathscr{R}^{1 \mid 1}\right)
$$

and

$$
\pi_{j}:\left(\mathbf{R}^{m}, \mathscr{R}^{m \mid m}\right) \rightarrow\left(\mathbf{R}, \mathscr{R}^{1 \mid 1}\right)
$$

is the projection onto the $j$ th factor $(1 \leq j \leq m)$. Then it is only natural to say that such a $\Phi$ is superlinear if and only if $\Phi_{j}$ is superlinear for each $j$. As an immediate consequence we obtain the following. 
3.7 COROLlaRY. $\Phi:\left(\mathbf{R}^{k}, \mathscr{R}^{k \mid k}\right) \rightarrow\left(\mathbf{R}^{m}, \mathscr{R}^{m \mid m}\right)$ is superlinear if and only if its coordinate expression is of the form

$$
\Phi^{\#}\left(\pi_{j}^{\#} t\right)=\sum A_{j i} p_{i}^{\#} t \quad \text { and } \quad \Phi^{\#}\left(\pi_{j}^{\#} \varsigma\right)=\sum A_{j i} p_{i}^{\#} \varsigma,
$$

where the $A_{j i}$ 's are real numbers and $\{t, \varsigma\}$ is any coordinate system on $\mathbf{R}^{1 \mid 1}$.

To close this section, let us point out that if $\Phi:\left(\mathbf{R}^{k}, \mathscr{R}^{k \mid k}\right) \rightarrow\left(\mathbf{R}^{m}, \mathscr{R}^{m \mid m}\right)$ is superlinear, then we can use this result and proceed as in 3.1 to prove that, for any $f \in C^{\infty}\left(\mathbf{R}^{m}\right)$,

$$
\Phi^{\#} f=f \circ \tilde{\Phi}
$$

where $\tilde{\Phi}: \mathbf{R}^{k} \rightarrow \mathbf{R}^{m}$ is the linear map whose matrix with respect to the coordinates $\left\{p_{i}^{\#} t\right\}$ in $\mathbf{R}^{k}$ and $\left\{\pi_{j}^{\#} t\right\}$ in $\mathbf{R}^{m}$ is just $\left(A_{j i}\right)$ as it appears in the corollary above. In particular, superlinear maps come from vector bundle maps; i.e., $\Phi^{\#}$ maps the odd coordinates of $\mathbf{R}^{m \mid m}$ into the odd coordinates of $\mathbf{R}^{k \mid k}$ in a linear fashion and (3.16) says that $\Phi^{\#}$ is in fact a $C^{\infty}\left(\mathbf{R}^{m}\right)$-module morphism. This is to be contrasted with the morphism $\mu_{k}: \mathbf{R}^{1 \mid 1} \times \mathbf{R}^{k \mid k} \rightarrow \mathbf{R}^{k \mid k}$ defined in (3.4) which, according to 3.4(ii), will never be a $C^{\infty}\left(\mathbf{R}^{k}\right)$-module map.

4. Superbilinearity. In this section we shall show that the notion of bilinearity within the category of supermanifolds gives rise to nontrivial supermanifold morphisms in the sense that they are not just maps of vector bundles. The supermanifolds we shall be dealing with are the same as before; namely, supermanifoldifications of real supervector spaces.

Just as in the case of superlinearity, the notion of superbilinearity is defined in terms of an abstract supermanifold morphism, $B: \mathbf{R}^{k \mid k} \times \mathbf{R}^{k \mid k} \rightarrow \mathbf{R}^{1 \mid 1}$ say, having the property of making some appropriate diagrams commute (see (4.9) and (4.10), which are the analogues of equations (3.10)). In order to state the results of this section we must introduce, in addition to the notation in (3.4)-(3.7),

$$
\chi_{j}: \mathbf{R}^{4 k \mid 4 k} \rightarrow \mathbf{R}^{1 \mid 1},
$$

which denotes the projection of the product of the $4 k$ copies of $\mathbf{R}^{1 \mid 1}$ onto the $j$ th factor $(1 \leq j \leq 4 k)$. We shall also introduce the morphisms

$$
\eta: \mathbf{R}^{4 k \mid 4 k} \rightarrow \mathbf{R}^{2 k \mid 2 k} \text { and } \lambda: \mathbf{R}^{4 k \mid 4 k} \rightarrow \mathbf{R}^{2 k \mid 2 k},
$$

which are completely determined by the conditions

$$
\begin{aligned}
\tau_{i} & \circ \eta=\chi_{i}, \quad \tau_{i} \circ \lambda=\chi_{2 k+i}, \quad 0 \leq i, j \leq k . \\
\tau_{k+j} & \circ \eta=\chi_{k+j}, \quad \tau_{k+j} \circ \lambda=\chi_{3 k+j}, \quad
\end{aligned}
$$

Therefore, we may consider the morphism

$$
\sigma_{k} \circ \eta \times \sigma_{k} \circ \lambda: \mathbf{R}^{4 k \mid 4 k} \rightarrow \mathbf{R}^{2 k \mid 2 k}
$$

so that

$$
\begin{aligned}
& \tau_{i} \circ\left(\sigma_{k} \circ \eta \times \sigma_{k} \circ \lambda\right)=s \circ\left(\chi_{i} \times \chi_{k+i}\right), \\
& \tau_{k+j} \circ\left(\sigma_{k} \circ \eta \times \sigma_{k} \circ \lambda\right)=s \circ\left(\chi_{2 k+j} \times \chi_{3 k+j}\right),
\end{aligned}
$$

On the other hand, we define the morphisms

$$
\begin{aligned}
& \alpha: \mathbf{R}^{4 k \mid 4 k} \rightarrow \mathbf{R}^{2 k \mid 2 k}, \quad \beta: \mathbf{R}^{4 k \mid 4 k} \rightarrow \mathbf{R}^{2 k \mid 2 k}, \\
& \gamma: \mathbf{R}^{4 k \mid 4 k} \rightarrow \mathbf{R}^{2 k \mid 2 k}, \quad \delta: \mathbf{R}^{4 k \mid 4 k} \rightarrow \mathbf{R}^{2 k \mid 2 k}
\end{aligned}
$$


by means of the conditions

$$
\begin{aligned}
& \tau_{i} \circ \alpha=\chi_{i}, \quad \tau_{i} \circ \beta=\chi_{i}, \quad \tau_{i} \circ \gamma=\chi_{k+i}, \quad \tau_{i} \circ \delta=\chi_{k+i}, \\
& \tau_{k+j} \circ \alpha=\chi_{2 k+j}, \quad \tau_{k+j} \circ \beta=\chi_{3 k+j}, \quad \tau_{k+j} \circ \gamma=\chi_{2 k+j}, \quad \tau_{k+j} \circ \delta=\chi_{3 k+j} \text {, } \\
& 0 \leq i, j \leq k \text {. }
\end{aligned}
$$

Now, the condition for a supermanifold morphism

$$
B: \mathbf{R}^{k \mid k} \times \mathbf{R}^{k \mid k} \rightarrow \mathbf{R}^{1 \mid 1}
$$

to be (super-) biadditive is expressed by means of the equation

$$
B \circ\left(\sigma_{k} \circ \eta \times \sigma_{k} \circ \lambda\right)=s \circ\{s \circ(B \circ \alpha \times B \circ \beta) \times s \circ(B \circ \gamma \times B \circ \delta)\}
$$

and we have the following.

4.1 Proposition. If $B: \mathbf{R}^{k \mid k} \times \mathbf{R}^{k \mid k} \rightarrow \mathbf{R}^{1 \mid 1}$ is a biadditive supermanifold morphism, then its coordinate expression in terms of an arbitrary coordinate system $\{t, \varsigma\}$ on $\mathbf{R}^{1 \mid 1}$ is given by

$$
\begin{aligned}
& B^{\#} t=\sum_{i} \sum_{j} B_{i j} \tau_{i}^{\#} t \tau_{k+j}^{\#} t+\sum_{i} \sum_{j} C_{i j} \tau_{i}^{\#} \varsigma \tau_{k+j}^{\#} \varsigma, \\
& B^{\#} \varsigma=\sum_{i} \sum_{j} D_{i j} \tau_{i}^{\#} \varsigma \tau_{k+j}^{\#} t+\sum_{i} \sum_{j} E_{i j} \tau_{i}^{\#} t \tau_{k+j}^{\#} \varsigma,
\end{aligned}
$$

where $B_{i j}, C_{i j}, D_{i j}$, and $E_{i j}$ are real constants and the summations take place over $1 \leq i \leq k$ and $1 \leq j \leq k$.

ProOF. We proceed in several steps. First, we write $B^{\#} t$ and $B^{\#} \zeta$ in the form

$$
\begin{aligned}
& B^{\#} t=b_{0}+\sum_{\mu<\nu} b_{\mu \nu} \tau_{\mu}^{\#} \varsigma \tau_{\nu}^{\#} \varsigma+\mathcal{J}^{4}\left(\mathbf{R}^{2 k \mid 2 k}\right), \\
& B^{\#} \varsigma=\sum_{\mu} b_{\mu} \tau_{\mu}^{\#} \varsigma+\sum_{\lambda<\mu<\nu} b_{\lambda \mu \nu} \tau_{\lambda}^{\#} \varsigma \tau_{\mu}^{\#} \varsigma \tau_{\nu}^{\#} \varsigma+\mathcal{J}^{5}\left(\mathbf{R}^{2 k \mid 2 k}\right),
\end{aligned}
$$

where $b_{0}, b_{\mu}, b_{\mu \nu}, b_{\lambda \mu \nu}, \ldots$ are $C^{\infty}$ functions on $\mathbf{R}^{2 k}$.

CONVENTION. Throughout this proof, greek indices run from 1 to $2 k$, while latin indices run from 1 to $k$. In passing from one type of indexation to the other we let

$$
U=\operatorname{Span}\left\{\tau_{\mu}^{\#} \varsigma: 1 \leq \mu \leq k\right\} \quad \text { and } \quad W=\operatorname{Span}\left\{\tau_{\mu}^{\#} \varsigma: k+1 \leq \mu \leq k\right\}
$$

and we use the decomposition

$$
\begin{aligned}
\bigwedge^{p}(U \oplus W) \simeq & \bigwedge^{p}(U) \otimes \mathbf{R} \oplus \bigwedge^{p-1}(U) \otimes \bigwedge^{1}(W) \\
& \oplus \cdots \oplus \bigwedge^{1}(U) \otimes \bigwedge^{p-1}(W) \oplus \mathbf{R} \otimes \bigwedge^{p}(W) .
\end{aligned}
$$

Thus, for example, the second-order terms $\sum_{\mu<\nu} b_{\mu \nu} \tau_{\mu}^{\#} \varsigma \tau_{\nu}^{\#} \varsigma$ will be written in the form

$$
\sum_{i<j} b_{i j} \tau_{i}^{\#} \varsigma \tau_{j}^{\#} \varsigma+\sum_{i} \sum_{j} b_{i, k+j} \tau_{i}^{\#} \varsigma \tau_{k+j}^{\#} \varsigma+\sum_{i<j} b_{k+i, k+j} \tau_{k+i}^{\#} \varsigma \tau_{k+j}^{\#} \varsigma .
$$


Let us now look at the left-hand side of (4.9) when we take the pull-back of the even coordinate $t$. According to our previous results in 3.4 and (4.1)-(4.5), we have

$$
\begin{aligned}
& {\left[B \circ\left(\sigma_{k} \circ \eta \times \sigma_{k} \circ \lambda\right)\right]^{\#} t=\left(\sigma_{k} \circ \eta \times \sigma_{k} \circ \lambda\right)^{\#} B^{\#} t} \\
& =\left(\sigma_{k} \circ \eta \times \sigma_{k} \circ \lambda\right)^{\#}\left(b_{0}+\sum_{\mu<\nu} b_{\mu \nu} \tau_{\mu}^{\#} \varsigma \tau_{\nu}^{\#} \varsigma+\mathcal{J}^{4}\left(\mathbf{R}^{2 k \mid 2 k}\right)\right) \\
& =\left(\sigma_{k} \circ \eta \times \sigma_{k} \circ \lambda\right)^{\#}\left(b_{0}\right) \\
& +\left(\sigma_{k} \circ \eta \times \sigma_{k} \circ \lambda\right)^{\#}\left(\sum_{i<j} b_{i j} \tau_{i}^{\#} \varsigma \tau_{j}^{\#} \varsigma\right) \\
& +\left(\sigma_{k} \circ \eta \times \sigma_{k} \circ \lambda\right)^{\#}\left(\sum_{i} \sum_{j} b_{i, k+j} \tau_{i}^{\#} \varsigma \tau_{k+j}^{\#} \varsigma\right) \\
& +\left(\sigma_{k} \circ \eta \times \sigma_{k} \circ \lambda\right)^{\#}\left(\sum_{i<j} b_{k+i, k+j} \tau_{k+i}^{\#} \varsigma \tau_{k+j}^{\#} \varsigma\right)+\mathscr{J}^{4}\left(\mathbf{R}^{4 k \mid 4 k}\right) \\
& =\left(\sigma_{k} \circ \eta \times \sigma_{k} \circ \lambda\right)^{\#}\left(b_{0}\right) \\
& +\sum_{i<j}\left(\sigma_{k} \circ \eta \times \sigma_{k} \circ \lambda\right)^{\#}\left(b_{i j}\right)\left[\chi_{i}^{\#} \varsigma+\chi_{k+i}^{\#} \varsigma\right]\left[\chi_{j}^{\#} \varsigma+\chi_{k+j}^{\#} \varsigma\right] \\
& +\sum_{i} \sum_{j}\left(\sigma_{k} \circ \eta \times \sigma_{k} \circ \lambda\right)^{\#}\left(b_{i, k+j}\right)\left[\chi_{i}^{\#} \varsigma+\chi_{k+i}^{\#} \varsigma\right]\left[\chi_{2 k+j}^{\#} \varsigma+\chi_{3 k+j}^{\#} \varsigma\right] \\
& +\sum_{i<j}\left(\sigma_{k} \circ \eta \times \sigma_{k} \circ \lambda\right)^{\#}\left(b_{k+i, k+j}\right) \\
& \times\left[\chi_{2 k+i}^{\#} \varsigma+\chi_{3 k+i}^{\#} \varsigma\right]\left[\chi_{2 k+j}^{\#} \varsigma+\chi_{3 k+j}^{\#} \varsigma\right]+\mathcal{J}^{4}\left(\mathbf{R}^{4 k \mid 4 k}\right) .
\end{aligned}
$$

On the other hand, when we take the pull-back of $t$, the right-hand side of (4.9) yields

$$
\begin{aligned}
& {[s \circ\{s \circ(B \circ \alpha \times B \circ \beta) \times s \circ(B \circ \gamma \times B \circ \delta)\}]^{\#} t} \\
& =\alpha^{\#} B^{\#} t+\beta^{\#} B^{\#} t+\gamma^{\#} B^{\#} t+\delta^{\#} B^{\#} t \\
& =\alpha^{\#} b_{0}+\beta^{\#} b_{0}+\gamma^{\#} b_{0}+\delta^{\#} b_{0} \\
& +\alpha^{\#}\left(\sum_{i<j} b_{i j} \tau_{i}^{\#} \varsigma \tau_{j}^{\#} \varsigma+\sum_{i} \sum_{j} b_{i, k+j} \tau_{i}^{\#} \varsigma \tau_{k+j}^{\#} \varsigma\right. \\
& \left.+\sum_{i<j} b_{k+i, k+j} \tau_{k+i}^{\#} \varsigma \tau_{k+j}^{\#} \varsigma\right) \\
& +\beta^{\#}\left(\sum_{i<j} b_{i j} \tau_{i}^{\#} \varsigma \tau_{j}^{\#} \varsigma+\sum_{i} \sum_{j} b_{i, k+j} \tau_{i}^{\#} \varsigma \tau_{k+j}^{\#} \varsigma\right. \\
& \left.+\sum_{i<j} b_{k+i, k+j} \tau_{k+i}^{\#} \varsigma \tau_{k+j}^{\#} \varsigma\right)
\end{aligned}
$$


(continued)

$$
\begin{aligned}
& +\gamma^{\#}\left(\sum_{i<j} b_{i j} \tau_{i}^{\#} \varsigma \tau_{j}^{\#} \varsigma+\sum_{i} \sum_{j} b_{i, k+j} \tau_{i}^{\#} \varsigma \tau_{k+j}^{\#} \varsigma\right. \\
& \left.+\sum_{i<j} b_{k+i, k+j} \tau_{k+i}^{\#} \varsigma \tau_{k+j}^{\#} \varsigma\right) \\
& +\delta^{\#}\left(\sum_{i<j} b_{i j} \tau_{i}^{\#} \varsigma \tau_{j}^{\#} \varsigma+\sum_{i} \sum_{j} b_{i, k+j} \tau_{i}^{\#} \varsigma \tau_{k+j}^{\#} \varsigma\right. \\
& \left.+\sum_{i<j} b_{k+i, k+j} \tau_{k+i}^{\#} \varsigma \tau_{k+j}^{\#} \varsigma\right) \\
& +\mathscr{J}^{4}\left(\mathbf{R}^{4 k \mid 4 k}\right) \\
& =\alpha^{\#} b_{0}+\beta^{\#} b_{0}+\gamma^{\#} b_{0}+\delta^{\#} b_{0} \\
& +\sum_{i<j}\left\{\alpha^{\#}\left(b_{i j}\right)+\beta^{\#}\left(b_{i j}\right)\right\} \chi_{i}^{\#} \varsigma \chi_{j}^{\#} \varsigma \\
& +\sum_{i<j}\left\{\gamma^{\#}\left(b_{i j}\right)+\delta^{\#}\left(b_{i j}\right)\right\} \chi_{k+i}^{\#} \varsigma \chi_{k+j}^{\#} \varsigma \\
& +\sum_{i<j}\left\{\alpha^{\#}\left(b_{k+i, k+j}\right)+\gamma^{\#}\left(b_{k+i, k+j}\right)\right\} \chi_{2 k+i}^{\#} \varsigma \chi_{2 k+j}^{\#} \varsigma \\
& +\sum_{i<j}\left\{\beta^{\#}\left(b_{k+i, k+j}\right)+\delta^{\#}\left(b_{k+i, k+j}\right)\right\} \chi_{3 k+i}^{\#} \varsigma \chi_{3 k+j}^{\#} \varsigma \\
& +\sum_{i} \sum_{j} \alpha^{\#}\left(b_{i, k+j}\right) \chi_{i}^{\#} \varsigma \chi_{2 k+j}^{\#} \varsigma+\sum_{i} \sum_{j} \beta^{\#}\left(b_{i, k+j}\right) \chi_{i}^{\#} \varsigma \chi_{3 k+j}^{\#} \varsigma \\
& +\sum_{i} \sum_{j} \gamma^{\#}\left(b_{i, k+j}\right) \chi_{k+i}^{\#} \varsigma \chi_{2 k+j}^{\#} \varsigma \\
& +\sum_{i} \sum_{j} \delta^{\#}\left(b_{i, k+j}\right) \chi_{k+i}^{\#} \varsigma \chi_{3 k+j}^{\#} \varsigma+\mathcal{J}^{4}\left(\mathbf{R}^{4 k \mid 4 k}\right)
\end{aligned}
$$

where we have used (4.6) and (4.7). Now, comparison of the two expressions gives, for the zeroth-order terms,

$$
\left(\sigma_{k} \circ \eta \times \sigma_{k} \circ \lambda\right)^{\#}\left(b_{0}\right)=\alpha^{\#} b_{0}+\beta^{\#} b_{0}+\gamma^{\#} b_{0}+\delta^{\#} b_{0} .
$$

That is, $b_{0}$ is biadditive in the usual sense. With respect to the coordinates $\left\{\tau_{i}^{\#} t, \tau_{k+i}^{\#} t\right\}$ of $\mathbf{R}^{k} \times \mathbf{R}^{k}$ this means that

$$
b_{0}=\sum_{i} \sum_{j} B_{i j} \tau_{i}^{\#} t \tau_{k+j}^{\#} t, \quad B_{i j} \in \mathbf{R},
$$

where $b_{0}$ in the left-hand side really stands for the pull-back of $t$ under $b_{0}$ in the sense of the $C^{\infty}$ category. 
Similarly, upon comparison of the second-order terms, we obtain

$$
\begin{array}{ll}
\chi_{i}^{\#} \varsigma \chi_{j}^{\#} \varsigma \text { term: } & \left(\sigma_{k} \circ \eta \times \sigma_{k} \circ \lambda\right)^{\#}\left(b_{i j}\right)=\alpha^{\#}\left(b_{i j}\right)+\beta^{\#}\left(b_{i j}\right), \\
\chi_{i}^{\#} \varsigma \chi_{k+j}^{\#} \varsigma \text { term: } & \left(\sigma_{k} \circ \eta \times \sigma_{k} \circ \lambda\right)^{\#}\left(b_{i j}\right)=0, \\
\chi_{k+i}^{\#} \varsigma \chi_{k+j}^{\#} \varsigma \text { term: } & \left(\sigma_{k} \circ \eta \times \sigma_{k} \circ \lambda\right)^{\#}\left(b_{i j}\right)=\gamma^{\#}\left(b_{i j}\right)+\delta^{\#}\left(b_{i j}\right), \\
\chi_{i}^{\#} \varsigma \chi_{2 k+j}^{\#} \varsigma \text { term: } & \left(\sigma_{k} \circ \eta \times \sigma_{k} \circ \lambda\right)^{\#}\left(b_{i, k+j}\right)=\alpha^{\#}\left(b_{i, k+j}\right), \\
\chi_{i}^{\#} \varsigma \chi_{3 k+j}^{\#} \varsigma \text { term: } & \left(\sigma_{k} \circ \eta \times \sigma_{k} \circ \lambda\right)^{\#}\left(b_{i, k+j}\right)=\beta^{\#}\left(b_{i, k+j}\right), \\
\chi_{k+i}^{\#} \varsigma \chi_{2 k+j}^{\#} \varsigma \text { term: } & \left(\sigma_{k} \circ \eta \times \sigma_{k} \circ \lambda\right)^{\#}\left(b_{i, k+j}\right)=\gamma^{\#}\left(b_{i, k+j}\right), \\
\chi_{k+i}^{\#} \varsigma \chi_{3 k+j}^{\#} \varsigma \text { term: } & \left(\sigma_{k} \circ \eta \times \sigma_{k} \circ \lambda\right)^{\#}\left(b_{i, k+j}\right)=\delta^{\#}\left(b_{i, k+j}\right), \\
\chi_{2 k+i}^{\#} \varsigma \chi_{2 k+j}^{\#} \varsigma \text { term: } & \left(\sigma_{k} \circ \eta \times \sigma_{k} \circ \lambda\right)^{\#}\left(b_{k+i, k+j}\right) \\
& =\alpha^{\#}\left(b_{k+i, k+j}\right)+\gamma^{\#}\left(b_{k+i, k+j}\right), \\
\chi_{2 k+i}^{\#} \varsigma \chi_{3 k+j}^{\#} \varsigma \text { term: } & \left(\sigma_{k} \circ \eta \times \sigma_{k} \circ \lambda\right)^{\#}\left(b_{k+i, k+j}\right)=0, \\
\chi_{3 k+i}^{\#} \varsigma \chi_{3 k+j}^{\#} \varsigma \text { term: } & \left(\sigma_{k} \circ \eta \times \sigma_{k} \circ \lambda\right)^{\#}\left(b_{k+i, k+j}\right) \\
& =\beta^{\#}\left(b_{k+i, k+j}\right)+\delta^{\#}\left(b_{k+i, k+j}\right),
\end{array}
$$

from which we conclude, by an argument similar to the one used in the proof of 3.4 , that

$$
b_{i j}=0 ; \quad b_{i, k+j}=\text { constant }=C_{i j} ; \quad b_{k+i, k+j}=0 .
$$

All together, this means that

$$
B^{\#} t=\sum_{i} \sum_{j} B_{i j} \tau_{i}^{\#} t \tau_{k+j}^{\#} t+\sum_{i} \sum_{j} C_{i j} \tau_{i}^{\#} \varsigma \tau_{k+j}^{\#} \varsigma+\mathcal{J}^{4}\left(\mathbf{R}^{2 k \mid 2 k}\right), \quad B_{i j}, C_{i j} \in \mathbf{R} .
$$

The rest of the argument proceeds inductively so as to prove that $B^{\#} t$ can have no component along $\mathcal{J}^{2 s}\left(\mathbf{R}^{2 k \mid 2 k}\right)$ for $s \geq 2$. In fact, application of $\left(\sigma_{k} \circ \eta \times \sigma_{k} \circ \lambda\right) \#$ to $B^{\#} t$ will result in $2^{2 s}$ different components in

$$
C^{\infty}\left(\mathbf{R}^{2 k} \times \mathbf{R}^{2 k}\right) \oplus \bigwedge^{2 s}(U \oplus W \times U \oplus W)
$$

out of each of its original $C^{\infty}\left(\mathbf{R}^{2 k}\right) \otimes \Lambda^{2 s}(U \oplus W)$ components. On the other hand, when we apply $\alpha^{\#}, \beta^{\#}, \gamma^{\#}$ and $\delta^{\#}$ successively to $B^{\#} t$ and add the resulting expressions up we find that only four different components are obtained in $C^{\infty}\left(\mathbf{R}^{2 k} \times \mathbf{R}^{2 k}\right) \otimes \bigwedge^{2 s}(U \oplus W \times U \oplus W)$ out of each of the original ones. Therefore, after demanding the equality of the $2 s$ th-order terms, we find that as soon as $s \geq 1$, the pull-back under the smooth map $\left(\sigma_{k} \circ \eta \times \sigma_{k} \circ \lambda\right) \sim$ of each original coefficient vanishes. Hence, the coefficients themselves must vanish.

A similar argument may be applied to the odd section $B^{\#} \varsigma$ to conclude that it can have no $\mathcal{J}^{2 s+1}\left(\mathbf{R}^{2 k \mid 2 k}\right)$ component for $s \geq 1$. Thus,

$$
B^{\#} \varsigma=\sum_{\mu} b_{\mu} \tau_{\mu}^{\#} \varsigma=\sum_{i} b_{i} \tau_{i}^{\#} \varsigma+\sum_{j} b_{k+j} \tau_{k+j}^{\#} \varsigma
$$


Therefore, when we apply $\left(\sigma_{k} \circ \eta \times \sigma_{k} \circ \lambda\right)^{\#}$ we get

$$
\begin{aligned}
\left(\sigma_{k} \circ \eta \times \sigma_{k} \circ \lambda\right)^{\#} B^{\#} \varsigma= & \left(\sigma_{k} \circ \eta \times \sigma_{k} \circ \lambda\right)^{\#}\left(\sum_{i} b_{i} \tau_{i}^{\#} \varsigma+\sum_{j} b_{k+j} \tau_{k+j}^{\#} \varsigma\right) \\
= & \sum_{i}\left(\sigma_{k} \circ \eta \times \sigma_{k} \circ \lambda\right)^{\#}\left(b_{i}\right)\left[\chi_{i}^{\#} \varsigma+\chi_{k+i}^{\#} \varsigma\right] \\
& +\sum_{j}\left(\sigma_{k} \circ \eta \times \sigma_{k} \circ \lambda\right)^{\#}\left(b_{k+j}\right)\left[\chi_{2 k+j}^{\#} \varsigma+\chi_{3 k+j}^{\#} \varsigma\right] .
\end{aligned}
$$

On the other hand,

$$
\begin{aligned}
\alpha^{\#} B^{\#} \varsigma & +\beta^{\#} B^{\#} \varsigma+\gamma^{\#} B^{\#} \varsigma+\delta^{\#} B^{\#} \varsigma \\
= & \alpha^{\#}\left(\sum_{i} b_{i} \tau_{i}^{\#} \varsigma+\sum_{j} b_{k+j} \tau_{k+j}^{\#} \varsigma\right)+\beta^{\#}\left(\sum_{i} b_{i} \tau_{i}^{\#} \varsigma+\sum_{j} b_{k+j} \tau_{k+j}^{\#} \varsigma\right) \\
& +\gamma^{\#}\left(\sum_{i} b_{i} \tau_{i}^{\#} \varsigma+\sum_{j} b_{k+j} \tau_{k+j}^{\#} \varsigma\right)+\delta^{\#}\left(\sum_{i} b_{i} \tau_{i}^{\#} \varsigma+\sum_{j} b_{k+j} \tau_{k+j}^{\#} \varsigma\right) \\
= & \sum_{i}\left[\alpha^{\#}\left(b_{i}\right)+\beta^{\#}\left(b_{i}\right)\right] \chi_{i}^{\#} \varsigma+\sum_{i}\left[\gamma^{\#}\left(b_{i}\right)+\delta^{\#}\left(b_{i}\right)\right] \chi_{k+i}^{\#} \varsigma \\
& +\sum_{j}\left[\alpha^{\#}\left(b_{k+j}\right)+\gamma^{\#}\left(b_{k+j}\right)\right] \chi_{2 k+j}^{\#} \varsigma+\sum_{j}\left[\beta^{\#}\left(b_{k+j}\right)+\delta^{\#}\left(b_{k+j}\right)\right] \chi_{3 k+j}^{\#} \varsigma .
\end{aligned}
$$

Therefore, we conclude that

$$
\begin{gathered}
\alpha^{\#}\left(b_{i}\right)+\beta^{\#}\left(b_{i}\right)=\left(\sigma_{k} \circ \eta \times \sigma_{k} \circ \lambda\right)^{\#}\left(b_{i}\right)=\gamma^{\#}\left(b_{i}\right)+\delta^{\#}\left(b_{i}\right), \\
\alpha^{\#}\left(b_{k+j}\right)+\gamma^{\#}\left(b_{k+j}\right)=\left(\sigma_{k} \circ \eta \times \sigma_{k} \circ \lambda\right)^{\#}\left(b_{k+j}\right)=\beta^{\#}\left(b_{k+j}\right)+\delta^{\#}\left(b_{k+j}\right) .
\end{gathered}
$$

Now, $b_{i}$ and $b_{k+j}$ are $C^{\infty}$ functions on $\mathbf{R}^{k} \times \mathbf{R}^{k}$ and we can apply the methods of the proofs in $\S 3$ : then the first set of equalities imply that $b_{i}$ must be independent of its first $k$ arguments and must be additive as a function of the $\tau_{k+j}^{\#} t$ coordinates $(1 \leq j \leq k)$. Similarly, the second set of equalities imply that $b_{k+j}$ is independent of the $\left(\tau_{k+j}^{\#} t\right)$ 's $(1 \leq j \leq k)$ and must be additive in the $\left(\tau_{i}^{\#} t\right)$ 's $(1 \leq i \leq k)$. Thus,

$$
b_{i}=\sum_{j} D_{i j} \tau_{k+j}^{\#} t \quad \text { and } \quad b_{k+j}=\sum_{i} E_{i j} \tau_{i}^{\#} t, \quad D_{i j}, E_{i j} \in \mathbf{R}
$$

which completes the proof.

Just as in the superlinear case, the homogeneity property (i.e., commutativity of the given morphism with superscalar multiplication) will impose further relations among the real coefficients appearing in the statement of 4.1. More precisely, we have the following.

4.2 PROPOSITION. Let $B: \mathbf{R}^{k \mid k} \times \mathbf{R}^{k \mid k} \rightarrow \mathbf{R}^{1 \mid 1}$ be a biadditive supermanifold morphism which in addition satisfies the homogeneity condition

$$
B \circ\left(\mu_{k} \times \operatorname{id}_{\mathbf{R}^{k \mid k}}\right)=m \circ\left(\operatorname{id}_{\mathbf{R}^{1 \mid 1}} \times B\right)
$$


(understood as morphisms from $\mathbf{R}^{1 \mid 1} \times \mathbf{R}^{k \mid k} \times \mathbf{R}^{k \mid k}$ into $\mathbf{R}^{1 \mid 1}$ ). Then its coordinate expression in terms of an arbitrary coordinate system $\{t, \zeta\}$ on $\mathbf{R}^{1 \mid 1}$ is given by

$$
\begin{aligned}
& B^{\#} t=\sum_{i} \sum_{j} B_{i j} \tau_{i}^{\#} t \tau_{k+j}^{\#} t+\sum_{i} \sum_{j} E_{i j} \tau_{i}^{\#} \varsigma \tau_{k+j}^{\#} \varsigma, \\
& B^{\#} \varsigma=\sum_{i} \sum_{j} B_{i j} \tau_{i}^{\#} \varsigma \tau_{k+j}^{\#} t+\sum_{i} \sum_{j} E_{i j} \tau_{i}^{\#} t \tau_{k+j}^{\#} \varsigma,
\end{aligned}
$$

where the $B_{i j}$ 's and the $E_{i j}$ 's are real constants and the summations take place over $1 \leq i \leq k$ and $1 \leq j \leq k$.

PROOF. The proof is a straightforward computation based on the result 4.1; this time the details shall be left to the reader.

4.3 REMARK. Let us note that there are various ways of stating the homogeneity condition on the second entry; in fact, this depends on the various symmetry requirements we demand from the given morphism $B$. The condition for $B$ to be supersymmetric, however, cannot be stated in terms of the commutativity of any diagram of supermanifold morphisms. The reason goes back to the fact that there is no way of stating the supercommutativity property of the supermultiplication morphism $m: \mathbf{R}^{1 \mid 1} \times \mathbf{R}^{1 \mid 1} \rightarrow \mathbf{R}^{1 \mid 1}$; more precisely, there is no supermanifold morphism $\varphi: \mathbf{R}^{1 \mid 1} \rightarrow \mathbf{R}^{1 \mid 1}$ for which the following diagram commutes:

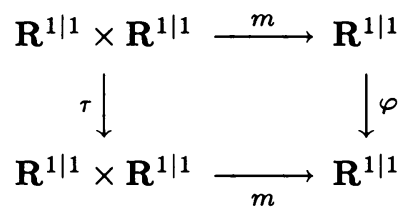

where $\tau: \mathbf{R}^{1 \mid 1} \times \mathbf{R}^{1 \mid 1} \rightarrow \mathbf{R}^{1 \mid 1} \times \mathbf{R}^{1 \mid 1}$ denotes the twist morphism, uniquely defined via the pair of conditions $p_{1} \circ \tau=p_{2}$ and $p_{2} \circ \tau=p_{1} ; p_{i}: \mathbf{R}^{1 \mid 1} \times \mathbf{R}^{1 \mid \mathbf{1}} \rightarrow \mathbf{R}^{\mathbf{1} \mid \mathbf{1}}$ being the projection of the product onto the ith factor $(i=1,2)$.

This result, which the reader can easily check for himself, is somewhat analogous to the fact that complex conjugation is not a holomorphic map. (By the way, this is the reason why we have said before that the morphisms (3.2) endow $\mathbf{R}^{1 \mid 1}$ with the structure of an abstract superalgebra; that is, we have omitted the word supercommutative.) We can, nevertheless, try to guess what the supersymmetry condition should translate into in terms of the $B_{i j}$ 's and the $E_{i j}$ 's appearing in 4.2 : following the rules of linear superalgebra and the results of $\S 2$, we would divide the even coordinates $\tau_{i}^{\#} t, 1 \leq i \leq k$, into the first $p$ (denoted $\tau_{i}^{\#} t, 1 \leq i \leq p$ ) and the last $q$ (denoted $\tau_{\pi i}^{\#} t, p+1 \leq i \leq p+q$ ), where $p+q=k$ and it is assumed that $\mathbf{R}^{k \mid k}$ was obtained as the result of the supermanifoldification of a supervector space of dimension $(p, q)$. A similar distinction is made among the odd coordinates; namely, the first $p$ (denoted $\tau_{\pi i}^{\#} \varsigma, 1 \leq i \leq p$ ) and the last $q$ (denoted $\tau_{i}^{\#} \varsigma, p+1 \leq i \leq p+q$ ). Then the supersymmetry conditions are

$$
B_{i j}=E_{\pi i, \pi j}, \quad B_{\pi i, \pi j}=-E_{i j}, \quad B_{\pi i, j}=-E_{i, \pi j}, \quad B_{i, \pi j}=E_{\pi i, j}
$$

On the other hand, and for the sake of comparison, we may impose a symmetry condition on $B$ expressible in terms of the commutativity of a certain diagram. 
For example, one possibility - which is closely related to the supersymmetric onewould be

$$
B \circ\left(\operatorname{id}_{\mathbf{R}^{k \mid k}} \times \mu_{k}\right) \circ\left(\tau \times \mathrm{id}_{\mathbf{R}^{k \mid k}}\right)=m \circ\left(\operatorname{id}_{\mathbf{R}^{1 \mid 1}} \times B\right)
$$

where again, both sides are understood as morphisms from $\mathbf{R}^{1 \mid 1} \times \mathbf{R}^{k \mid k} \times \mathbf{R}^{k \mid k}$ into $\mathbf{R}^{1 \mid 1}$ and $\tau: \mathbf{R}^{1 \mid 1} \times \mathbf{R}^{k \mid k} \rightarrow \mathbf{R}^{k \mid k} \times \mathbf{R}^{1 \mid 1}$ denotes the twist morphism that permutes the factors. In this case, it is a straightforward matter to verify that the following holds true:

4.4 Proposition. Let $B: \mathbf{R}^{k \mid k} \times \mathbf{R}^{k \mid k} \rightarrow \mathbf{R}^{1 \mid 1}$ be a supermanifold morphism which, in addition to the hypotheses of 4.2, satisfies (4.12). Then its coordinate expression in terms of an arbitrary coordinate system $\{t, \zeta\}$ on $\mathbf{R}^{1 \mid 1}$ is given by

$$
\begin{aligned}
& B^{\#} t=\sum_{i} \sum_{j} B_{i j} \tau_{i}^{\#} t \tau_{k+j}^{\#} t+\sum_{i} \sum_{j} B_{i j} \tau_{i}^{\#} \varsigma \tau_{k+j}^{\#} \varsigma, \\
& B^{\#} \varsigma=\sum_{i} \sum_{j} B_{i j} \tau_{i}^{\#} \varsigma \tau_{k+j}^{\#} t+\sum_{i} \sum_{j} B_{i j} \tau_{i}^{\#} t \tau_{k+j}^{\#} \varsigma,
\end{aligned}
$$

where the $B_{i j}$ 's are real constants and the summations take place over $1 \leq i \leq k$ and $1 \leq j \leq k$. In other words, $B$ is completely determined by its underlying (continuous) bilinear form $\tilde{B}$.

From now on, we shall refer to supermanifold morphisms $B: \mathbf{R}^{k \mid k} \times \mathbf{R}^{k \mid k} \rightarrow \mathbf{R}^{1 \mid 1}$ satisfying the hypotheses of 4.2 as superbilinear. Let us note that superbilinear morphisms are not vector bundle maps. In fact, even though $B^{\#} \varsigma$ is given in 4.2 as a $C^{\infty}\left(\mathbf{R}^{2 k}\right)$-linear combination of the odd coordinates in $\mathbf{R}^{k \mid k} \times \mathbf{R}^{k \mid k}$, the expression for $B^{\#} t$ shows that, for any $f \in C^{\infty}(\mathbf{R})$,

$$
B^{\#} f=f \circ \tilde{B}+\sum_{i} \sum_{j}\left(f^{\prime} \circ \tilde{B}\right) B_{i j} \tau_{i}^{\#} \varsigma \tau_{k+j}^{\#} \varsigma,
$$

where $\tilde{B}$ is the underlying bilinear map $\mathbf{R}^{k} \times \mathbf{R}^{k} \rightarrow \mathbf{R}$ of $B$ whose matrix, with respect to the coordinate system $\left\{\tau_{i}^{\#} t ; \tau_{k+j}^{\#} t\right\}$, is $\left(B_{i j}\right)$; the notation being as in 4.2 .

To close this section, let us briefly indicate how our results have to be modified in order to incorporate superbilinear morphisms of the form

$$
\Phi: \mathbf{R}^{k \mid k} \times \mathbf{R}^{m \mid m} \rightarrow \mathbf{R}^{1 \mid 1}
$$

and more generally, those of the form

$$
\Psi: \mathbf{R}^{k \mid k} \times \mathbf{R}^{m \mid m} \rightarrow \mathbf{R}^{n \mid n}
$$

obtained as $n$-tuples $\left(\Psi_{1}, \Psi_{2}, \ldots, \Psi_{n}\right)$ of the type (4.14) with $\Psi_{h}=\pi_{h} \circ \Psi ; \pi_{h}$ being the projection morphism $\mathbf{R}^{n \mid n} \rightarrow \mathbf{R}^{1 \mid 1}$ onto the $h$ th factor $(1 \leq h \leq n)$. We claim that all that has to be done is to recognize that $\Phi$ as in (4.14) will be superbilinear if and only if it satisfies the pair of conditions

$$
\begin{gathered}
\Phi \circ\left(\sigma_{k} \circ \eta \times \sigma_{k} \circ \lambda\right)=s \circ\{s \circ(\Phi \circ \alpha \times \Phi \circ \beta) \times s \circ(\Phi \circ \gamma \times \Phi \circ \delta)\}, \\
\Phi \circ\left(\mu_{k} \times \operatorname{id}_{\mathbf{R}^{k \mid k}}\right)=m \circ\left(\operatorname{id}_{\mathbf{R}^{1 \mid 1}} \times \Phi\right),
\end{gathered}
$$

where now $\eta, \lambda, \alpha, \beta, \gamma$ and $\delta$ are the supermanifold morphisms

$$
\begin{gathered}
\eta: \mathbf{R}^{2 k \mid 2 k} \times \mathbf{R}^{2 m \mid 2 m} \rightarrow \mathbf{R}^{2 k \mid 2 k}, \quad \lambda: \mathbf{R}^{2 k \mid 2 k} \times \mathbf{R}^{2 m \mid 2 m} \rightarrow \mathbf{R}^{2 m \mid 2 m}, \\
\alpha, \beta, \gamma, \delta: \mathbf{R}^{2 k \mid 2 k} \times \mathbf{R}^{2 m \mid 2 m} \rightarrow \mathbf{R}^{k \mid k} \times \mathbf{R}^{m \mid m}
\end{gathered}
$$


defined as in (4.3) and (4.7) with the only difference that this time $1 \leq i \leq k$ and $1 \leq j \leq m$. The natural generalization of 4.2 is then

4.5 Proposition. Let $\Psi: \mathbf{R}^{k \mid k} \times \mathbf{R}^{m \mid m} \rightarrow \mathbf{R}^{n \mid n}$ be such that for each $h$ $(1 \leq h \leq n)$, the supermanifold morphism $\Psi_{h}=\pi_{h} \circ \Psi: \mathbf{R}^{k \mid k} \times \mathbf{R}^{m \mid m} \rightarrow \mathbf{R}^{1 \mid 1}$ is superbilinear in the sense above. Then its coordinate expression in terms of an arbitrary coordinate system $\{t, \varsigma\}$ on $\mathbf{R}^{1 \mid 1}$ is given by

$$
\begin{aligned}
& \Psi^{\#} \pi_{h}^{\#} t=\sum_{i} \sum_{j} C_{h i j} \tau_{i}^{\#} t \tau_{k+j}^{\#} t+\sum_{i} \sum_{j} D_{h i j} \tau_{i}^{\#} \varsigma \tau_{k+j}^{\#} \varsigma, \\
& \Psi^{\#} \pi_{h}^{\#} \varsigma=\sum_{i} \sum_{j} C_{h i j} \tau_{i}^{\#} \varsigma \tau_{k+j}^{\#} t+\sum_{i} \sum_{j} D_{h i j} \tau_{i}^{\#} t \tau_{k+j}^{\#} \varsigma,
\end{aligned}
$$

where the $C_{h i j}$ 's and the $D_{h i j}$ 's are real constants and the summations take place over $1 \leq i \leq k$ and $1 \leq j \leq m$.

The example we are particularly interested in is the one that results when

(i) $\mathbf{R}^{m \mid m}$ is viewed as the supermanifoldification of the real $(p, q)$-dimensional supervector space $V=V_{0} \oplus V_{1}$, with $m=p+q$,

(ii) $\mathbf{R}^{n \mid n}$ is viewed as the supermanifoldification of the real $(r, s)$-dimensional supervector space $W=W_{0} \oplus W_{1}$, with $n=r+s$, and

(iii) $\mathbf{R}^{k \mid k}$ is then viewed as the supermanifoldification of $\operatorname{Hom}(V, W)$.

What comes out from this example in the light of 4.5 are the various superbilinear pairings $\operatorname{Hom}(V, W)_{S} \times V_{S} \rightarrow W_{S}$. This observation brings us back to the problem stated in $\S 2$ of finding, among the bilinear morphisms (in the category of supermanifolds), a suitable generalization of the action (or evaluation) map. What we now see is that the heuristic approach followed in $\S 2$ yields precisely an example of such a superbilinear morphism.

\section{BIBLIOGRAPHY}

[Kostant] B. Kostant, Graded manifolds, graded Lie theory and prequantization, Lecture Notes in Math., vol. 570, Springer-Verlag, Berlin and New York, 1977.

[LeYtes] D. Leĭtes, Introduction to the theory of supermanifolds, Russian Math. Surveys 35 (1980), $1-64$.

[Manin] Yu. Manin, Holomorphic supergeometry and Yang-Mills superfields (preprint).

[OASV1] O. A. Sánchez Valenzuela, Matrix computations in linear superalgebra, Linear Algebra Appl. (to appear).

[OASV2] _ _ On supervector bundles (preprint).

Instituto de Investigaciones en Matemáticas Aplicadas y Sistemas, Universidad nacional Autónoma de México, Delegacion Alvaro Obregón, 01000, MÉXICO D.F.

Current address: Centro de Investigación en Matematicas, Apartado Postal 402, C.P. 3600, Guanajuato, Gto, Mexico 